\title{
The Polyhedral Model of Nonlinear Loops
}

ARAVIND SUKUMARAN-RAJAM and PHILIPPE CLAUSS, INRIA, Team CAMUS, ICube Lab, CNRS, University of Strasbourg, France

Runtime code optimization and speculative execution are becoming increasingly prominent to leverage performance in the current multi- and many-core era. However, a wider and more efficient use of such techniques is mainly hampered by the prohibitive time overhead induced by centralized data race detection, dynamic code behavior modeling, and code generation. Most of the existing Thread Level Speculation (TLS) systems rely on naively slicing the target loops into chunks and trying to execute the chunks in parallel with the help of a centralized performance-penalizing verification module that takes care of data races. Due to the lack of a data dependence model, these speculative systems are not capable of doing advanced transformations, and, more importantly, the chances of rollback are high. The polyhedral model is a wellknown mathematical model to analyze and optimize loop nests. The current state-of-art tools limit the application of the polyhedral model to static control codes. Thus, none of these tools can generally handle codes with while loops, indirect memory accesses, or pointers. Apollo (Automatic POLyhedral Loop Optimizer) is a framework that goes one step beyond and applies the polyhedral model dynamically by using TLS. Apollo can predict, at runtime, whether the codes are behaving linearly or not, and it applies polyhedral transformations on-the-fly. This article presents a novel system that enables Apollo to handle codes whose memory accesses and loop bounds are not necessarily linear. More generally, this approach expands the applicability of the polyhedral model at runtime to a wider class of codes. Plugging together both linear and nonlinear accesses to the dependence prediction model enables the application of polyhedral loop optimizing transformations even for nonlinear code kernels while also allowing a low-cost speculation verification.

Categories and Subject Descriptors: D.3.4 [Programming Languages]: Processors-Optimizations

General Terms: Algorithms, Performance

Additional Key Words and Phrases: Speculative and dynamic loop parallelization, polyhedral model, nonlinear memory references

ACM Reference Format:

Aravind Sukumaran-Rajam and Philippe Clauss. 2015. The polyhedral model of nonlinear loops. ACM Trans. Archit. Code Optim. 12, 4, Article 48 (December 2015), 27 pages.

DOI: http://dx.doi.org/10.1145/2838734

\section{INTRODUCTION}

With the current trend regarding microprocessors, execution-time performance is increasingly depending on software since it becomes mainly its own responsibility to take advantage of the available hardware computing resources. In particular, parallelization of applications is definitely crucial for the utilization of multicore processor architectures in addition to other important optimization issues such as data locality. However, parallel programming is generally complex, error prone, and cannot be handled easily by just any programmer. One has to reason about possible dependencies

Authors' addresses: A. Sukumaran-Rajam and P. Clauss, ICube Lab., 300 bd Sébastien Brant, CS 10413, F-67412 Illkirch Cedex; emails: \{philippe.clauss, aravind.sukumaran-rajam\}@inria.fr.

Permission to make digital or hard copies of part or all of this work for personal or classroom use is granted without fee provided that copies are not made or distributed for profit or commercial advantage and that copies show this notice on the first page or initial screen of a display along with the full citation. Copyrights for components of this work owned by others than ACM must be honored. Abstracting with credit is permitted. To copy otherwise, to republish, to post on servers, to redistribute to lists, or to use any component of this work in other works requires prior specific permission and/or a fee. Permissions may be requested from Publications Dept., ACM, Inc., 2 Penn Plaza, Suite 701, New York, NY 10121-0701 USA, fax +1 (212) 869-0481, or permissions@acm.org.

(c) 2015 ACM 1544-3566/2015/12-ART48 $\$ 15.00$

DOI: http://dx.doi.org/10.1145/2838734 
to avoid any data race while manually parallelizing the code. Moreover, if the dependencies depend on some input data or if parallelizing the code is only valid in certain phases of the execution, manual parallelization is usually not performed because it is extremely hard to handle such cases. In typical manual parallelization, advanced loop transformations are usually not applied. However, transformations are important because they may expose more parallelism and optimization opportunities. Thus, one may rely on automatic code optimizers and optimizing compilers that include automatic parallelization techniques of serial codes. But these techniques are still very limited in the kind of codes they can handle since, in general, important information regarding dependencies between program instructions and memory operations may only be known at runtime.

Static optimizers can only rely on the information that is available statically and thus have to make very conservative assumptions. A promising strategy for overcoming such limitations is to perform code optimization at runtime-while the target code is running -in order to take advantage of the dynamically available information. Runtime optimizers have two major advantages: One is that more information is available as the values of variables are resolved; the other is the opportunity to reason about the code behavior by observing it. Regarding runtime loop parallelization, several frameworks have been proposed based on Thread-Level Speculation (TLS) [Liu et al. 2006; Raman et al. 2008; Rauchwerger and Padua 1995], in which parallel execution of code regions is enabled before all dependencies are known. Hardware or software mechanisms track register and memory accesses to determine if any dependence violation-or data race-has occurred. In such cases, the register and memory state is rolled back to a previous valid state, and sequential re-execution is initiated.

Traditional TLS systems perform a simple, straightforward parallelization of loop nests by simply slicing the outermost loop into consecutive parallel threads [Johnson et al. 2007; Liu et al. 2006; Rauchwerger and Padua 1995]. Modest performance improvements have been reported due to limitations in the kind of optimized code that can be generated at runtime and due to the prohibitive time overhead induced by the data race detection system, which is mostly centralized. Rather than blindly parallelizing loops, a dependence prediction model would help in determining an efficient optimizing transformation. For example, more advanced parallelizing transformations of loops, or data locality optimizations, are not handled by such dynamic frameworks. Yet, such transformations are essential for obtaining good performance of parallel codes. On the other hand, the polyhedral model [Bondhugula et al. 2008; Feautrier and Lengauer 2011] is a proven mathematical model for loop optimization, although it is strongly limited to loop nests with static control, affine memory accesses, and loop bounds.

Existing loop optimizers that use the polyhedral model can only work on statically analyzable code. For a loop to be characterized as statically analyzable in the polyhedral model, one should be able to determine the control flow using only statically available information, and one should be able to express the memory accesses and loop bounds in terms of a statically determinable linear functions. The presence of a simple while loop or indirect memory access (e.g., a [b [i] ] or ptr $=$ ptr $->$ next), or even a simple pointer whose alias analysis information cannot be determined, will prevent the code from being optimized by these tools. In Jimborean et al. [2014]; SukumaranRajam et al. [2014], we proposed a framework called Apollo (Automatic POLyhedral Loop Optimizer) that implements a dynamic and speculative adaptation of the polyhedral model. Apollo is able to optimize and parallelize loop nests that cannot be handled at compile-time and that exhibit a linear behavior at runtime by using a decentralized verification of the speculation. Handling codes at runtime using the polyhedral model adds the additional challenges of efficiently performing live instrumentation, dependence analysis, scheduling, memory backup, and code generation. 
In this article, we go further by proposing a strategy to extend our framework to loop nests that exhibit a nonlinear behavior. This work targets loops whose accessed memory addresses and/or loop bounds cannot be expressed as affine functions of the enclosing loop iterators and loop parameters, neither at compile-time nor at runtime. Nonlinearity implies the uncertainty in predicting the memory addresses that will be accessed. This has a direct impact on dependence analysis, scheduling, memory backup, and speculation verification. Our proposal, which is based on a new dynamic dependence modeling of the target code, extends the applicability of the polyhedral model to non-linear loops.

Apollo's general scheme is the following. At compile-time, for each target loop nest of a program, a set of generic (incomplete) optimized code versions is generated. Each code version, named skeleton, is a template dedicated to a wide class of optimizing transformations of the target loop nest. At runtime, instrumentation by sampling provides the information required to build a dynamic dependence model, which is then used as a prediction for the rest of the execution. This model has been extended to include simultaneously linear and nonlinear information. Then, the code scheduler is invoked to obtain an optimizing transformation. Based on the properties of the selected transformation, a corresponding generic skeleton is selected. The skeleton is then instantiated with parameters that mold it to the corresponding final transformed code.

This article brings the following contributions:

-An adaptation of the polyhedral model to handle and optimize loops with nonlinear memory accesses;

-An adaptation of the polyhedral model to handle and optimize loops with nonlinear loop bounds;

-An adaptation of the polyhedral model to handle functions inside a loop body, including recursive functions;

-A speculation verification system consisting of collaborative distributed (decentralized) and non-distributed (centralized) components, which significantly reduces the communication overhead regarding traditional fully centralized verification;

-A smart backup system for recovery in case of bad speculation, with reduced time cost and memory requirements thanks to the modeling of the runtime code behavior; -A strategy providing flexibility and adaptation to the current behavior by applying suitable optimizations to distinct program's phases.

The article is organized as follows: Section 2 recalls the adaptation of the polyhedral model to its dynamic usage implemented in Apollo. Section 3 explains the general architecture of Apollo. Section 4 presents the extensions of the model to handle nonlinear memory accesses and loop bounds. Section 5 addresses some related works. Section 6 shows the experiments and the results obtained on a set of benchmark codes. Finally, conclusions are given in Section 7.

\section{THE POLYHEDRAL MODEL AND ITS APPLICABILITY}

The polyhedral model [Bondhugula et al. 2008; Feautrier and Lengauer 2011] has been proved to be a powerful mathematical and geometrical framework for analyzing and optimizing for-loop nests. The requirements are that (i) each loop iterates according to a unique index variable whose bounds are affine expressions of the enclosing loop indices, and (ii) the memory instructions that are handled are limited to access simple scalar variables or multidimensional array elements referenced using affine expressions on the enclosing loop indices. Such loop nests are analyzed precisely with respect to data dependencies that occur among the statements and across iterations. Thus, advanced optimizing transformations are proved to be semantically correct by preserving 
the dependencies of the original program. The loop nest optimizations (e.g., loop skewing, loop interchange) are linear transformations of the iteration domains that are represented geometrically as polyhedra. Each tuple of loop indices values is associated with an integer point contained in a polyhedron. The order in which the iterations are executed translates to the lexicographic order of the tuples.

Thus, transformations represent reorderings of the iterations and are defined as scheduling matrices, which are equivalent to geometrically transforming a polyhedron into another equivalent form. Representing loop nests as polyhedra enables one to reason about the valid transformations that can be performed.

\subsection{Limitations of the Polyhedral Model}

Although very powerful, the polyhedral model is restricted to a small class of computeand memory-intensive codes that can be analyzed accurately and transformed only at compile-time. However, most legacy codes are not amenable to this model due to indirect or pointer-based accesses to static or dynamic data structures that prevent a precise dependence analysis to be performed statically. Another common reason is nonaffine loop bounds. Function calls inside loop nests set another challenge to the polyhedral model because the function definition may not be available, which is essential to build the dependence polyhedron. Informally, it should be able to statically determine the exact memory accesses that will occur inside the function call.

For these reasons, even though the model is powerful, at present, the applicability is limited to very few code kernels. Whereas Pluto ${ }^{1}$ is considered the state-of-theart polyhedral compiler, it can only handle statically analyzable, affine-characterized codes.

On the other hand, if applied entirely dynamically, the complex analysis and the polyhedral code transformations would entail a significant time overhead. However, codes that do not exhibit characteristics suiting the polyhedral model at compile time may still be in compliance with the model, although this compliance can only be detected and validated at runtime. We call such codes dynamically polyhedral codes. Targeting such codes for automatic optimization and parallelization proposes to immerse the polyhedral model in the context of speculative and dynamic parallelization. In this context, runtime code analysis and transformation impose strategies that induce very low time overheads that must be largely compensated by the gains provided by the polyhedral optimization and parallelization.

\subsection{Dynamically Polyhedral Codes}

For the loop nests that cannot be analyzed statically but which exhibit a polyhedral behavior at runtime, our strategy for making the polyhedral model applicable relies on speculation coupled with runtime verification. It consists of observing initially the original code during a very short sample of the whole run (instrumentation by sampling). If a polyhedral behavior is observed on this sample, we speculate that the behavior will remain the same for the rest of the loop nest execution. Thus, we abstract the loop to a polyhedral representation, reason about the intra- and interiteration dependencies, and apply and validate polyhedral optimizations and parallelizing transformations. As long as the prediction remains true, the generated parallel code is semantically correct by definition of the polyhedral model. In order to continuously verify the prediction and thus verify the correctness of the speculatively optimized program, we implemented a decentralized runtime verification system embedded in the parallel code. The decentralized architecture, which is enabled thanks to the polyhedral model, has significantly less overhead when compared to a centralized system traditionally

\footnotetext{
$\overline{{ }^{1} \mathrm{http} / / / p l u t o-c o m p i l e r . s o u r c e f o r g e . n e t . ~}$
} 
used in TLS systems. This approach has been implemented in our Apollo framework [Jimborean et al. 2014; Sukumaran-Rajam et al. 2014].

Apollo consists of two main parts: a static part implemented as a set of passes of the LLVM compiler ${ }^{2}$ and a dynamic part implemented as a runtime system written in $\mathrm{C}++$.

At compile-time, Apollo's static phase (i) precisely analyzes memory instructions that can be disambiguated at compile-time and (ii) generates an instrumented version to track memory accesses that cannot be disambiguated at compile-time. The instrumented version is capable of running only on a sample of the outermost loop iterations and has mechanisms to pass the collected data to the runtime system. Next, (iii) it generates parallel code skeletons [Jimborean et al. 2014], which are incomplete versions of the transformed loop nest in the LLVM intermediate representation (LLVM-IR) and that require runtime instantiation for fast dynamic code generation. Each instantiation represents a new optimization; therefore, the code skeletons can be seen as highly generic templates that support a large set of optimizing and parallelizing transformations. Additionally, the skeletons embed support for speculation (e.g., verification). Adding generic support for transformations involves some overheads. Instead of using a single, very general skeleton that can support all the transformations, Apollo generates a set of skeletons, each supporting a different set of transformations. This choice allows Apollo to fine-tune the skeletons in such a way that the overhead that would have been issued from the support of nonselected transformations is completely eliminated. Moreover, runtime-instantiated skeletons in the LLVM-IR are finally translated into executable code using the LLVM Just-In-Time compiler, just before being effectively launched, thus being further optimized through traditional compiler optimization passes (constant propagation, common sub-expression elimination, dead code elimination, loop-invariant code motion, etc.).

At runtime, Apollo's dynamic phase (i) runs the instrumented version on a sample of consecutive outermost loop iterations, (ii) builds a linear prediction model for the loop bounds and memory accesses from the instrumentation data; (iii) computes dependencies between these accesses; (iv) selects and instantiates a code skeleton and generates an optimized parallel version of the original sequential code, semantically correct with respect to the prediction model, and which is going to run for a slice of the original outermost loop (also called a chunk); (v) backs up memory locations that are going to be modified, according to the prediction, during the execution of the next chunk; and (vi), during the execution of the multithreaded code, each thread verifies independently if the prediction still holds. If not, a rollback is initiated to recover the exection of last chunk, and the system attempts to build a new prediction model for the remaining future chunks.

\subsection{Nonlinear Codes}

Even if applied dynamically, the polyhedral model still limits the domain of codes that can be handled to those codes that exhibit a linear behavior, at least in some phases. This single factor eliminates a large class of codes because most codes have nonaffine loop bounds and nonaffine memory accesses. However, in this article, we show that the applicability of the model can be extended by amending certain runtime policies to accommodate these code classes and thanks to the speculative and dynamic context of application. Depending on the code kernel, the whole kernel or a part of it can be subjected to the effective optimizations provided by the polyhedral model, even if it does not exhibit a linear behavior.

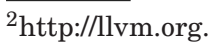




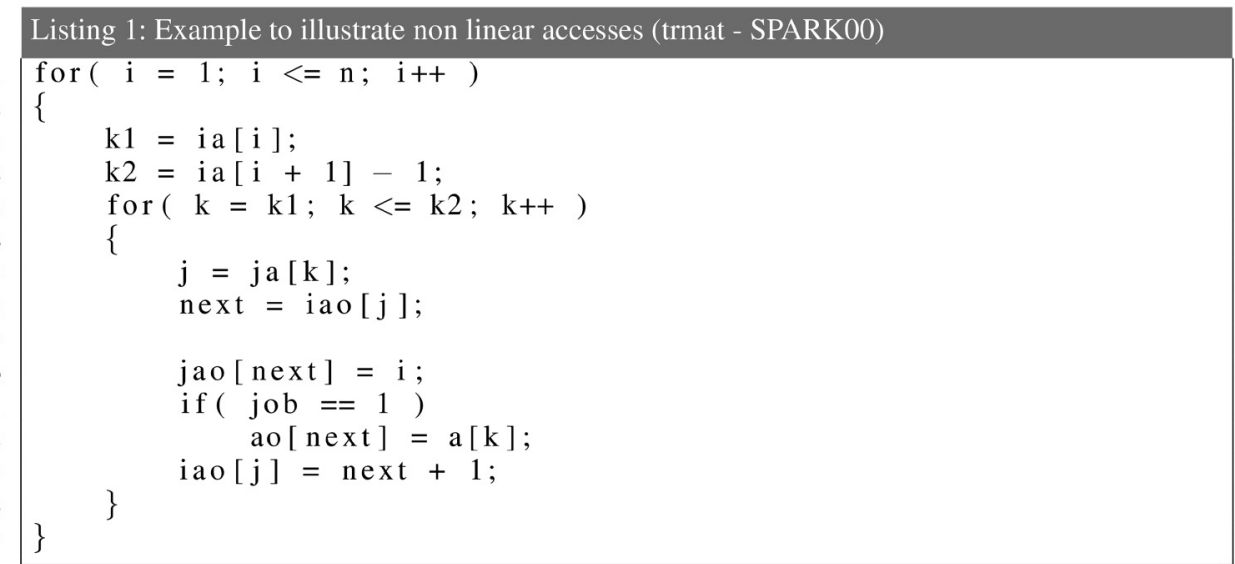

For a concrete example, consider the loop kernel of the trmat code from the SPARKOO benchmark suite [van der Spek et al. 2008] in Listing 1. This kernel computes the transpose of a sparse matrix. Since the matrix is sparse, the actual number of elements in each column is unknown. Hence, before executing this kernel, the benchmark code traverses the old index structure and populates the number of elements per column into array $i a$ (not shown in Listing 1). We would like to emphasize that this code cannot be handled statically using the polyhedral model for several reasons:

- the for-loop in Line 5 has bounds defined by values $k 1$ and $k 2$ that may change randomly during the execution of the loop nest since these are elements of array $i a$;

- a direct consequence is that the memory read $j a[k]$ in Line 7 cannot be disambiguated because values of loop iterator $k$ are unknown. The same holds for access $a[k]$ in Line 12;

- variable next holds the result of a single indirect access $i a o[j]$ in Line 8, which is actually $i a o[j a[k]]$. At runtime, this latter memory access may be linear, quasi-linear, or of any other kind. Similarly, the memory writes in Lines 10 and 12 both use double indirect accesses and hence may also be of any kind: Reference jao[next] is actually $j a o[i a o[j a[k]]]$, and reference $a o[n e x t]$ is actually ao[iao[ja[k]]].

To summarize, the code in Listing 1 has one loop with nonlinear loop bounds, and six memory accesses of unknown kinds; thus, seven unknown entities in total. It is worth noting that no existing polyhedral static tools can handle this code. The presence of just one unknown entity would force tools to choose the conservative approach of not optimizing this code. By using the techniques described in this article, however, we can successfully optimize such codes, even if they exhibit nonlinear memory behaviors and loop trip counts.

This article emphasizes techniques implemented in Apollo to handle such nonlinear behaviors. Table I shows a comparison that applies the polyhedral model statically among Pluto, Apollo, and the new Apollo with its extensions.

In the following section, we present an overview of the architecture of Apollo to show afterward how its flexibility enables it to also handle nonlinear codes. Further details regarding dynamic code generation and other important parts can be found in Jimborean et al. [2014] and Sukumaran-Rajam et al. [2014].

\section{ARCHITECTURE OF APOLLO}

Speculative parallelization and runtime verification are performed using the prediction model as follows: 
Table I. Comparison of Different Polyhedral Code Optimization Approaches

\begin{tabular}{|c|c|c|c|}
\hline Code Behavior & Pluto & Apollo & $\begin{array}{l}\text { NL } \\
\text { Apollo }\end{array}$ \\
\hline $\begin{array}{l}\text { Linear memory accesses, which are an affine function of loop iterators, } \\
\text { with constants and unknown loop parameters (e.g., } a[i+\mathrm{N}+\mathrm{c}])\end{array}$ & $\checkmark$ & $\checkmark$ & $\checkmark$ \\
\hline $\begin{array}{l}\text { Linear memory accesses, for which the loop iterator coefficients depend } \\
\text { on unknown loop parameters }(\text { e.g., } a[i * N+j])\end{array}$ & $x$ & $\checkmark$ & $\checkmark$ \\
\hline $\begin{array}{l}\text { Linear loop bounds, which are affine functions of loop iterators, } \\
\text { constants and unknown loop parameters }(\text { e.g., } \mathrm{j}<\mathrm{i}+\mathrm{N}+\mathrm{c})\end{array}$ & $\checkmark$ & $\checkmark$ & $\checkmark$ \\
\hline $\begin{array}{l}\text { Linear loop bounds, for which the loop iterator coefficients depend on } \\
\text { unknown loop parameters }(\text { e.g., } \mathrm{j}<\mathrm{i} * \mathrm{~N})\end{array}$ & $x$ & $\checkmark$ & $\checkmark$ \\
\hline $\begin{array}{l}\text { Statically unresolvable, dynamically linear memory accesses (e.g., } \\
\text { a[b[i]], where b[i] is linear at runtime) }\end{array}$ & $x$ & $\checkmark$ & $\checkmark$ \\
\hline $\begin{array}{l}\text { Statically unresolvable, dynamically linear memory accesses through } \\
\text { pointers (e.g., *p, where p is linear at runtime) }\end{array}$ & $x$ & $\checkmark$ & $\checkmark$ \\
\hline $\begin{array}{l}\text { Statically unresolvable, dynamically linear loop bounds (e.g., } \mathrm{j}<\mathrm{b}[\mathrm{i}] \text {, } \\
\text { where b[i] is linear at runtime) }\end{array}$ & $x$ & $\checkmark$ & $\checkmark$ \\
\hline Nonlinear read memory accesses & $x$ & $x$ & $\checkmark$ \\
\hline Nonlinear write memory accesses & $x$ & $x$ & $\checkmark$ \\
\hline Nonlinear loop bounds & $x$ & $x$ & $\checkmark$ \\
\hline Support for function calls (including recursion) inside the loop nest & $x$ & $x$ & $\checkmark$ \\
\hline
\end{tabular}

'a[]' and 'b[]' represent arrays, '*p' represents a memory access through a pointer, 'i' and 'j' are the loop iterators, 'c', a compile-time constant, and 'N' an unknown compile-time loop parameter.

Code skeletons. At compile-time, several variants of codes are generated from each loop nest that was marked in the source code by the user using a dedicated pragma that we added to the Clang-LLVM parser: This is an instrumented version, as described in the previous Section, but also a number of code skeletons, presented in detail in our previous paper [Jimborean et al. 2014]. Skeletons can be seen as parametrized codes, where the instantiation of their parameters result in the generation of a transformed optimized version of the target loop nest, merging original computations and speculative parallelization management. They consist of three parts (see Figure 1): The first part applies the transformation, which is populated at runtime; the second performs the original computation on the transformed iteration domain; and the third does the verification. Each skeleton supports a certain combination of loop transformations among skewing, interchange, tiling, and the like [Banerjee 1993]. In the current implementation, Apollo only supports skeletons for combinations of skewing, interchange, and parallelization. However, any other polyhedral transformation can be handled, subject to the implementation of additional corresponding skeleton generators in the LLVM compiler.

At compile time, various code characteristics such as loop structure, number of memory access statements and their access type, and other statically analyzable code properties are detected and encoded in the binary and thus are made available to the runtime.

Parallelizing code transformation. As soon as the prediction model has been built, Apollo's runtime system performs a dependence analysis that determines if the target loop nest can be parallelized and optimized and which transformation to be applied for this purpose. A polyhedral transformation merely refers to changing the order in which iterations are executed and is controlled by applying affine functions on the loop iterators. The transformation is encoded as a matrix, storing the coefficients of the affine functions that define the new schedule. For example and without loss of generality, given a loop nest of depth 2 with iterators $\left(\begin{array}{c}i \\ j\end{array}\right)$ and a transformation 


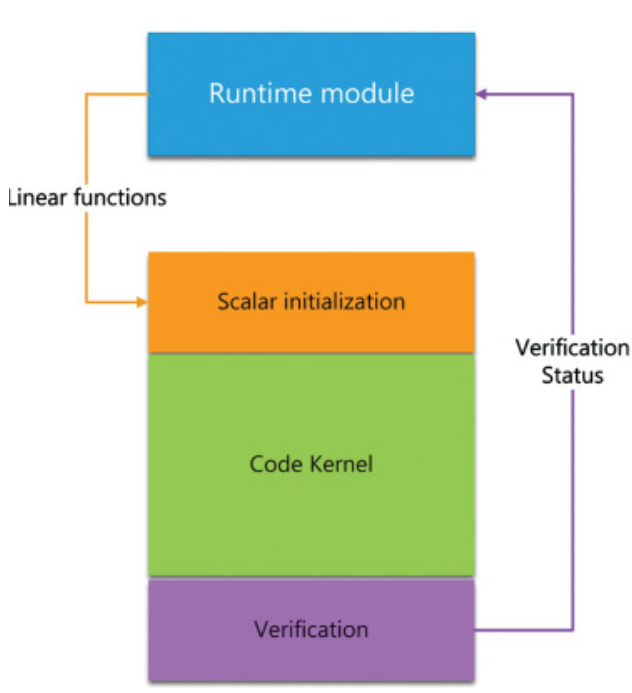

(a) Without nonlinear support

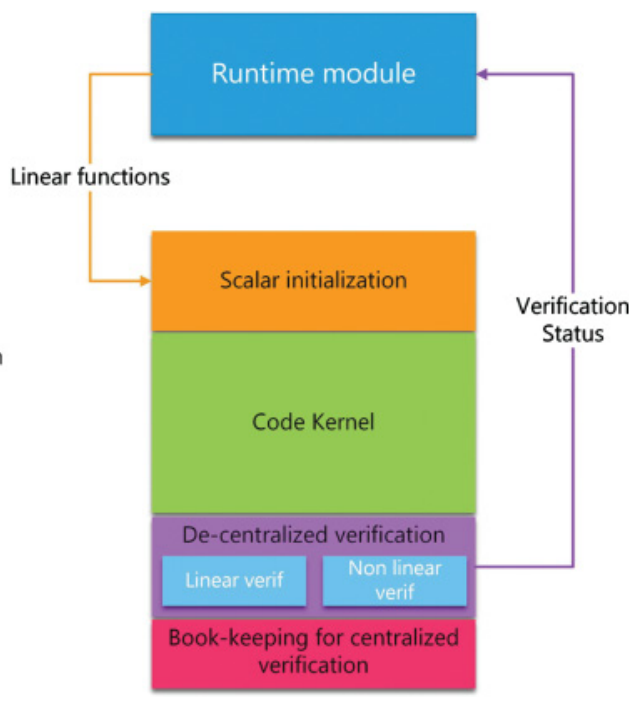

(b) With nonlinear support

Fig. 1. General structure of a skeleton.

matrix $T$, polyhedral loop transformations such as skewing, interchange, or any affine transformation of the iteration domains, are obtained as: $T \times\left(\begin{array}{c}i \\ j\end{array}\right)=\left(\begin{array}{c}i^{\prime} \\ j^{\prime}\end{array}\right)$.

Scheduling is achieved by invoking the Pluto polyhedral parallelizer at runtime. More precisely, only the scheduling kernel of Pluto is used by Apollo. It has been slightly customized to consume our dependence analysis output and to suggest a polyhedral transformation in return. Since Pluto aims simultaneously at data locality optimization and parallelization, the generated schedule is expected to lead to a well-performing parallel code. Notice also that Pluto is initially a source-to-source code compiler used at compile-time and that Apollo is the first known dynamic framework to use it at runtime.

Speculative code orchestration. The different code versions (instrumented, original sequential, or optimized skeletons) are launched into chunks of fixed sizes. Each chunk runs a slice of successive iterations of the outermost original loop nest. Thus, optimizing parallelizing transformations are applied on such slices. At startup, Apollo launches a small chunk running the instrumented version in order to build the prediction model, and it performs the dependence analysis. The transformation suggested by Pluto, using the dependence information, is then used to instantiate the code skeleton devoted to the corresponding class of transformations. The resulting parallel code is then launched inside a larger chunk, after having previously backed up the memory locations that are predicted to be updated. The verification code inside the skeleton will ensure that no unpredicted memory address will be modified. If the verification of the speculation detects an unpredicted behavior, memory is restored to cancel the execution of the current chunk. The execution of the chunk is reinitiated using the original sequential version in order to overcome the faulty execution point. Finally, an instrumented chunk is launched again to capture the changing behavior and to build a new prediction model. The rationale behind launching the instrumentation skeleton after a mis-prediction is to capture the new program phase and build a new dependence prediction model for the new phase. If no misprediction was detected during the run of the parallel code, the 


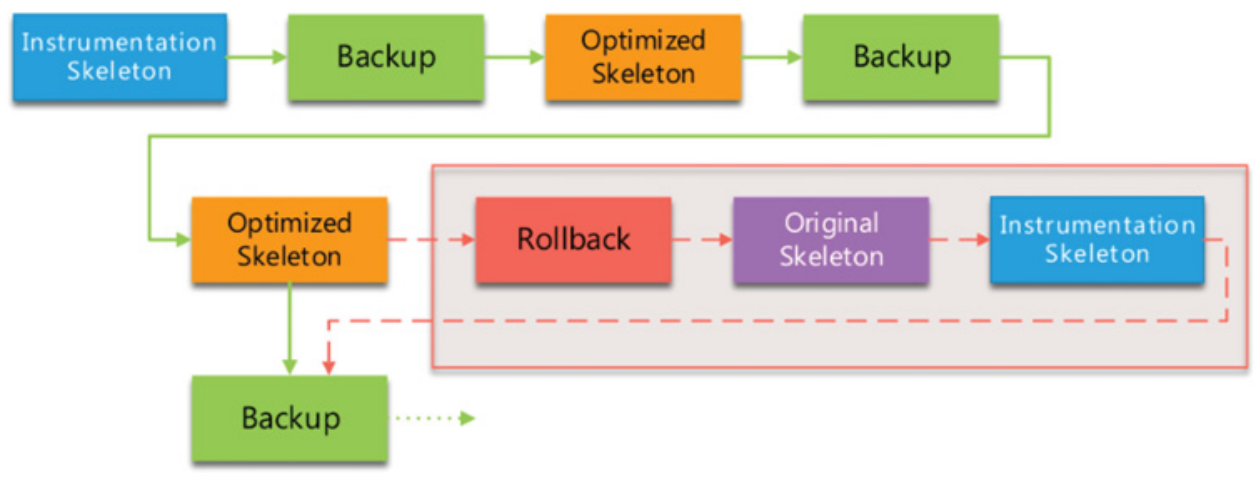

Fig. 2. Execution of the loop by chunks in Apollo.

next chunk is launched using the same parallel code. Figure 2 depicts this execution by chunks.

\section{EXTENDING THE POLYHEDRAL MODEL APPLICABILITY TO NONAFFINE LOOPS}

Indirect memory references and pointers are quite common in codes; hence, the ability to handle them is a prime challenge in automatic parallelization. Most of the codes exhibit nonlinear memory behavior or nonlinear loop bounds. Traditional TLS systems that may handle such codes require a huge amount of time for backing up memory while also incurring a huge time overhead from the centralized verification of the speculation. The lack of a prediction model results in higher chances for wrong parallelization and rollback. Moreover, additional loop transformations may be required to make the loop parallel and fast. Hence, a prediction model that can accurately represent the program behavior is essential for better performance.

However, even the presence of a single nonaffine entity inside a program invalidates the use of the polyhedral model (i.e., no polyhedral code transformation can be performed nor parallelization of any loop). The impact of this limitation prevents a lot of codes from being optimized even when most of their memory accesses are linear. This limitation arises because the dependence model cannot be constructed. When at least one nonaffine entity is present, the system should make conservative assumptions in order to guarantee correctness. The most conservative approach is obviously to execute the original code itself. In presence of a nonlinear entity, most polyhedral optimizers do so. In some very specific cases, some static checks can be encoded to still allow some optimizations, but, in general, this is not enough [Ravishankar et al. 2012].

To handle nonaffine entities, a general approach is to relax the dependence model. This can be done either by approximating the nonaffine accesses with affine functions, which can be computed by using linear regression, or by simply ignoring these accesses while constructing the dependence polyhedron and accounting for them later during verification. If the model is relaxed, then it no longer preserves the original guarantee on the validity of the optimizing transformation. This calls for a runtime validator in addition to the runtime linear verification system. Whether to populate the relaxed dependence model with regression equations or to ignore the nonaffine accesses depends on the quality of the regression hyperplanes regarding how they model the nonaffine accesses. The fitness of the regression hyperplanes regarding the original points can be expressed in terms of correlation coefficients [Cohen et al. 2002]. The correlation coefficient essentially measures the fitness of the predicted hyperplane and the observed points. If the correlation coefficient is high, then including the regression equation in the dependence model should provide a parallel schedule that optimizes the nonlinear 
accesses conjointly to the linear accesses. On the other hand, if the regression hyperplane has a low correlation coefficient, adding it to the dependence model may not provide any benefit with respect to optimizations; it could even be harmful to do so. In this latter case, it is better to optimize the schedule by initially ignoring the associated memory accesses. Additional runtime tests must then be performed to ensure subsequently the validity of the optimizing transformation.

In the following, we give a detailed description on how loops with nonaffine memory accesses and loop bounds are handled for speculative optimization and parallelization.

\subsection{Constructing the Dependence Polyhedron}

To predict the runtime code behavior, an initial instrumentation by sampling is performed. During the actual execution, a small slice of each loop of the target loop nest is profiled. The collected profile consists of the memory addresses that are accessed (possibly through successive function calls which may be recursive), the iteration counts, and the enclosing loop iterators. By analyzing the loop trip count of each loop and subjecting this information to linear interpolation, and if necessary, to linear regression, we can obtain the linear functions characterizing the loop bounds. Similarly, by analyzing the memory access information for each memory instruction, we can interpolate the linear functions characterizing the sequence of memory addresses that are accessed. The memory accesses occurring inside a function, possibly recursive and called from within the loop nest, are handled similarly to other memory accesses, although only the nonrecursive iterators are considered while constructing the linear functions. Essentially, all the memory accesses occurring inside a recursive function are treated as nonlinear memory accesses.

The fitness between the observed values of accessed memory addresses (or loop trip counts) is used to classify the accesses as either affine, nearly affine, or nonaffine. A memory access (or loop bound) is classified as:

—affine if all the observations fall perfectly into a linear equation and all the coefficients of the linear functions are integers;

-nearly affine if most of the observations stay "close" to a linear equation;

-nonaffine if the observations do not fall into the other two categories.

A two-dimensional graphical representation of these three cases is depicted in Figure 3. Accesses that can be completely modeled using linear equations couple perfectly with the polyhedral model. However, since the dependencies are calculated on the basis of a small instrumented sample, a runtime verification system is still required to validate the conformance of the prediction model to the rest of the code execution. The details of this verification system is presented in Sukumaran-Rajam et al. [2014]. Essentially, the verification system checks whether the predicted linear functions match with the actual execution.

The nearly affine behaviors are common in $\mathrm{C} / \mathrm{C}++$ programs that use malloc for allocating memory regions. Accesses to malloc-ed regions may not be affine due to various reasons, such as unavailability of contiguous space, differences between the access pattern and the allocation pattern, variation of the size for each allocation request either due to the program logic or due to security reasons: malloc may actually allocate more size than requested, so that buffer zones can be created, which greatly helps in avoiding buffer overflow attacks [Berger and Zorn 2006; Novark and Berger 2010]. Nonaffine accesses are usually the result of indirect accesses or of accesses through pointers where the program logic dictates accesses to memory locations in a random fashion.

As opposed to affine accesses, neither nearly affine nor nonaffine accesses can be naturally handled by the polyhedral model. In order to extend the scope of the model, the regression information must be expressed in a way that suits the model. The choice 

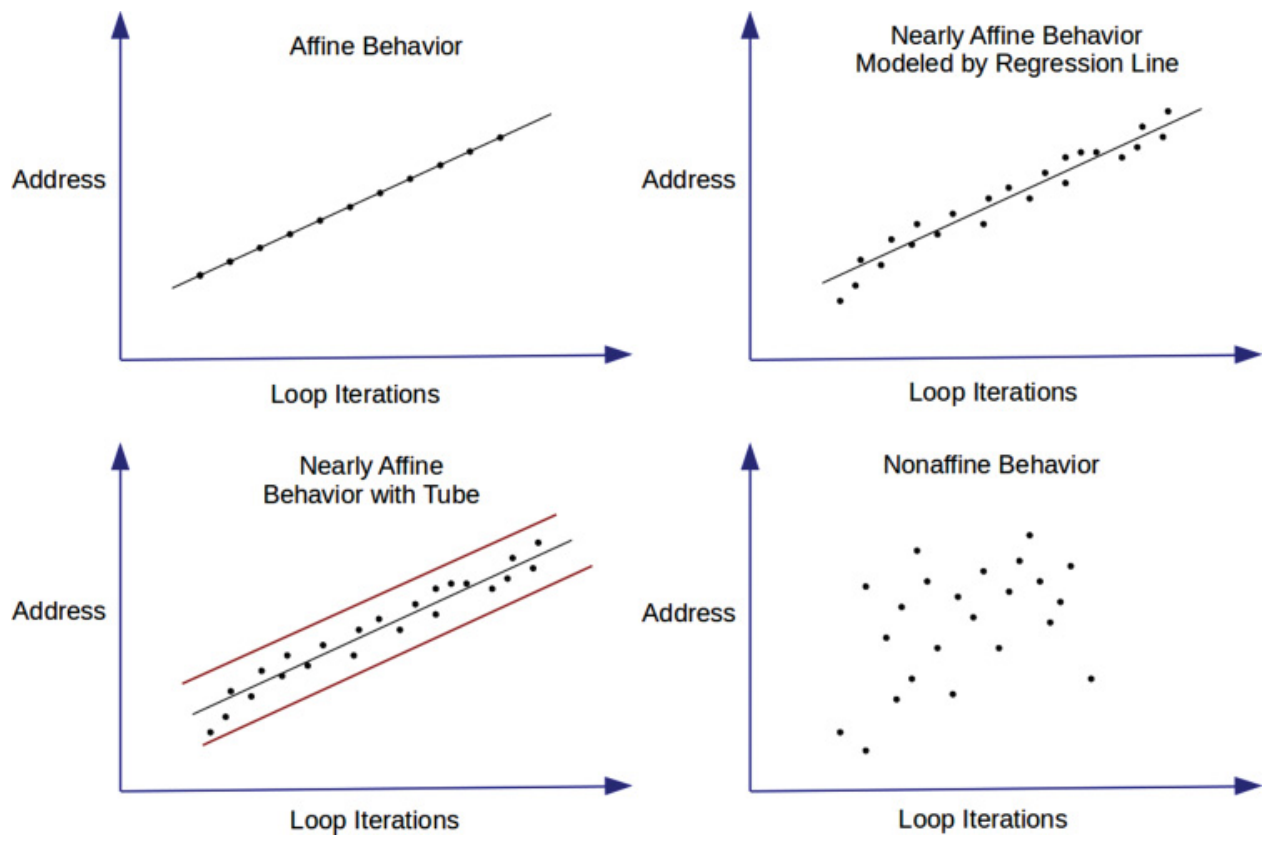

Fig. 3. A graphical representation of affine, nearly affine, and nonaffine behaviors.

of how memory accesses are characterized is based on the correlation coefficients of the regression hyperplanes. We distinguish two cases that are explained next.

\subsection{Nonlinear Memory Accesses Modeling}

Nonlinear memory accesses refer to those memory accesses (read or write) whose instances do not correspond to any affine function. Hence, a regression function is computed for such memory statements, and the associated correlation coefficient is computed. Based on its value, the nonlinear memory accesses are handled as follows.

\section{The correlation coefficient is between 0.9 and 1.}

A correlation coefficient between 0.9 and 1 indicates a good fit of the observed values with the regression equation and thus suggests that it well-approximates the actual memory accesses. Hence, it is reasonable to include this regression equation in the dependence model, the rationale being that the polyhedral optimizer (scheduler) gives a transformation that optimizes the nonaffine accesses conjointly with the affine accesses, thus optimizing data locality and parallelism for the affine and nonaffine accesses. The value 0.9 was selected as an optimal value due to the following reasons. If a higher value is selected, it will put more restrictions on the amount of nonlinearity. With a lower value, the amount of required backup will increase accordingly, resulting in less performance gains. Thus, 0.9 was selected since it provides a good balance that was observed through numerous experiments. It is worth noting that the correlation coefficient also represents the confidence of the linear function against the memory accesses. Thus, if the value is lower, it is better to treat the corresponding access using the range mechanism explained later.

Each regression hyperplane is computed by multivariate linear regression using the least-squares estimation [Cohen et al. 2002]. However, the presence of outlier points can sway the regression hyperplane away from the majority of the access points. To avoid this, we build the regression hyperplane in two steps: From an initial computed regression equation, we compute the distance of each point to the resulting regression 
hyperplane. Then, a set of points that are close to the median point is selected, and the regression hyperplane is recomputed by just considering these points. When compared to the original regression hyperplane, the newly constructed regression hyperplane provides a better fit to the memory accesses because the outliers were eliminated while computing it.

The polyhedral model requires the dependence constraints to be expressed in integer $\mathbb{Z}$-domains, whereas the regression hyperplanes lie in real $\mathbb{R}$-domains. For each regression hyperplane, the closest regression hyperplane in the $\mathbb{Z}$-domain is constructed by approximating the coefficients along each dimension to their closest integers. This approximation does not much alter the accuracy since the polyhedral model only considers the integer points that lie in the convex hull defined by the constraints.

The regression hyperplane should then be encoded into the dependence polyhedron. If encoded directly, like a linear function, the polyhedral scheduler only considers those points falling exactly on the regression hyperplane when computing the solution. Therefore, using only the regression equations is not sufficient because it ignores the points falling near the hyperplane. Hence, for each nearly affine access, two hyperplanes that are parallel to the regression hyperplane are constructed by adding positive and negative offsets to the original hyperplane. Informally, one hyperplane is "above" and the other is "below" the original regression hyperplane, both forming a tube around the regression hyperplane (see the lower left of Figure 3). The distance between the tubular hyperplanes (offset) is selected such that all the refined points fall inside the tube. This tube forming a convex hull of the observed points is then encoded into the dependence model.

\section{The correlation is less than 0.9 .}

If the correlation coefficient is less than 0.9 , then the memory accesses are characterized as non-affine and excluded from the dependence representation using regression. For each corresponding memory instruction, the accesses are then approximated using the range of the minimum and maximum values of the addresses that were accessed during instrumentation. Notice that this computed range may be very large. For example, consider a case in which the first loop iteration accesses the first element of a large array and the next one accesses the last element. In this case, it is better not to perform a range-based backup because the access is very sparse and the range size is very large (in this example, the whole array should be backed up). Apollo only preforms rangebased backup if the backup size is less than three times the expected backup size. The expected backup size is computed from the number of statement instances, which in turn can be deduced using the enclosing loop bounds. If the range-based backup is not performed, then all the accesses of the corresponding statement will be treated using a "live backup" mechanism (see Section 4.5).

Overlaps between such ranges, the regression tubes, and the linear functions are tested to know whether a dependence may occur, in which case no code transformation will be performed. This eager early detection of possible dependencies saves the time spent in backing up data, executing a code with an invalid transformation, and, finally, rolling back.

The inclusion of nearly affine and nonaffine accesses relaxes the polyhedral model but appends the challenge of handling additional verification of the memory accesses occurring outside the predicted regions. This is explained in Section 4.6.

\subsection{Nonlinear Loop Bounds Modeling}

In the presence of nonlinear loop bounds, the range of memory addresses that will be accessed cannot be determined even with dynamic instrumentation. Thus, we cannot build the dependence polyhedron. This is true even when all memory accesses are linear. 


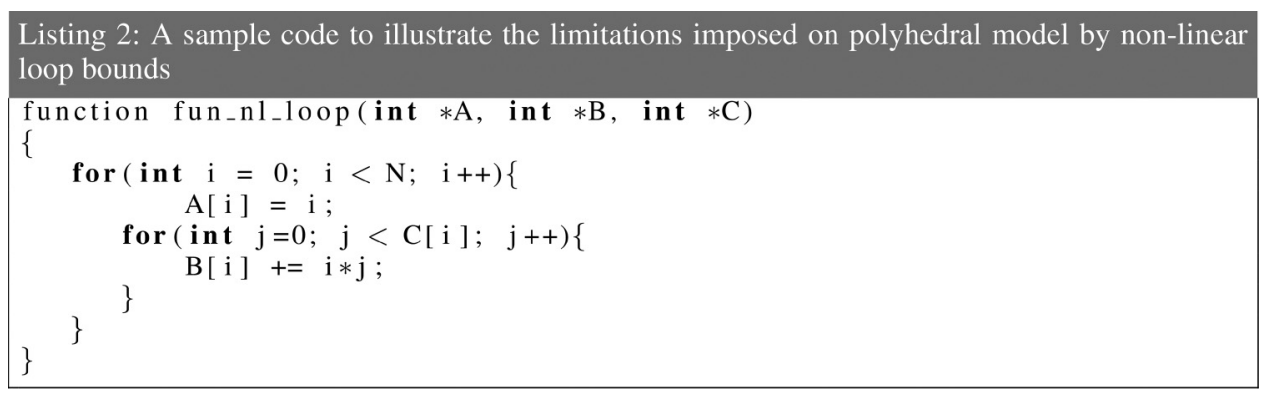

To illustrate this issue, consider the code in Listing 2 . The nonlinear inner j-loop has a bound based on the value of $\mathrm{C}[i]$. At runtime, if the successive values of $C$ [i] can be expressed as an affine function of iterator $i$ and loop parameter $\mathrm{N}$, then no extension is required for Apollo to handle this loop. Otherwise, by applying regression techniques and measuring the correlation coefficient, we can predict the values and build the model as explained next. It is worth noting that even though the memory access functions for all three arrays A, B, and C are linear, it is unknown at compile-time whether their address ranges overlap, or, in other words, whether they alias (e.g., if the function is invoked with (array1, array2, array 1) as arguments, then pointers A and $\mathrm{C}$ will alias). Compilers such as Pluto cannot handle this case due to the presence of nonlinear loop bounds. It is also worth noting that, even in the absence of nonlinear loop bounds, Pluto will assume that arrays A, B, and C do not alias, since it is the programmer's responsibility to ensure this; in Apollo, this is automatically verified.

Modeling a loop in the polyhedral model requires:

(1) A linear function for the lower bound of a loop, parametrized by its outer loop iterators, loop parameter, and constants;

(2) A linear function for the loop iterator, parametrized by its outer loop iterators, loop parameter, and constants;

(3) A constant representing the iterator increment;

(4) A linear function for the upper bound of a loop, parametrized by its outer loop iterators, loop parameter, and constants.

Items (1), (2), and (3) are determined simultaneously by instrumenting the scalar values characterizing the loop iterator. Item (4) is determined by instrumenting the loop trip count. For a given vector representing the values of the enclosing loop iterators, (1) and (2) are used to compute the current value of the iterator of the loop in consideration and (4) is used to compute until when the loop should iterate. The transformation framework relies on these linear functions to define the domain of the statements and the exact instances of the statements that are scanned.

For simplicity, consider only loop parallelization as being the optimizing transformation. Also assume that Items (1), (2) and (3) are at least dynamically linear (i.e., all the linear functions characterizing the loop, except the upper bound, were obtained by interpolation). Direct usage of a regression hyperplane may lead to an under- or overapproximated domain. This implies that a loop with nonlinear bounds cannot be parallelized directly. One approach to solve this issue would be to back up the addresses accessed by each loop just prior to its execution (note that Apollo does not do this; it only backs up regions based on the outermost loop, before the optimized skeleton is invoked), parallelize code in chunks, and execute the chunks one after the other until a rollback occurs. Then, restore the state and execute the last chunk sequentially. The immediate problem with this approach is the backup. Since each loop backs up data, it would increase the storage requirement exponentially, and there 
may be a lot of overlaps among backed-up data. Moreover, the control of the loop would be very complex, as well as the mechanism to restore memory.

We go one step further to enable parallelization of such loops. By computing a minimal hyperplane for the loop upper bounds, we expect all the loops to execute at least until the point where the iterator meets this hyperplane. If the minimal hyperplane holds its validity during execution, the nonlinear loop can be parallelized until this minimal hyperplane. Note that the parallelization should still be legal (i.e., the dependencies should not prevent parallelization). The remaining iterations of the nonlinear loop should be executed sequentially until it reaches the original loop exit condition. The advantage of this approach is that we are able to parallelize and optimize the predicted part with a very low time overhead. However, this does not guarantee the program correctness because the sequential part, which was not predicted, could introduce an unseen dependence, thus violating the dependence prediction model. The memory accesses occurring in the unpredicted space are therefore considered to be equivalent to nonlinear memory accesses. As explained in the previous section, Apollo's nonlinear memory access handling system can detect any violation on unpredicted memory regions and thus guarantees the program validity. If, during the actual execution, the loop runs iterations that are not predicted using the minimum hyperplane while the loop is marked for parallel execution, the system performs a rollback.

A similar approach to that of the upper bound can be followed for the lower bound. By using the current instance of the enclosing loop iterators and using regression, one can predict the lower loop bound. To handle nonlinearity, the loop can be run sequentially using the actual values until the predicted lower bound, and then it continues in a parallel manner. Both the upper and lower bound nonlinear techniques can be combined to produce three waves of computation: a head sequential part, a middle (core) parallel part, and a tail sequential part. Handling any transformation such as loop interchange, tiling, and the like follows the same principles.

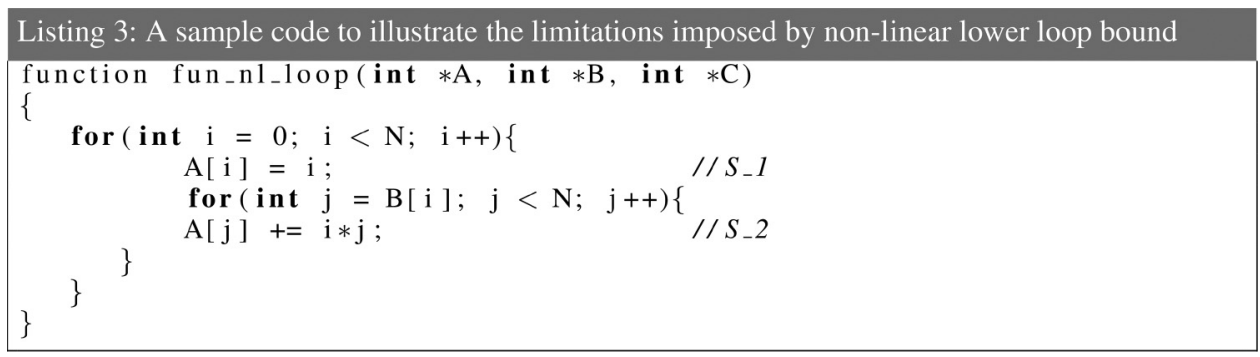

All the loop iterators appear as scalars in the code. The scalar prediction is thus responsible for the initialization of these values. If the loop lower bound cannot be predicted, the initialization of these values is impossible. Thus, these loops cannot be optimized using the polyhedral model. This is illustrated by the code example in Listing 3. The lower bound of the j-loop is determined by $B$ [i]. If the successive values of $B$ [i] do not follow a linear function, there is no way to initialize $j$ at each $i$-iteration. The nonlinear lower bound for loop $j$ can be treated as a memory address, and a related dependence can be added to the model. Effectively, for each i-loop iteration, the lower bound of the $\mathrm{j}$-loop is loaded, not predicted. The immediate consequence is that many optimizations are prevented. Hence, we choose another approach. Based on the correlation coefficient of the loop trip counts, the loops are processed as follows.

\section{The correlation coefficient is between 0.9 and 1 .}

A high value of the correlation coefficient indicates that the loop bounds tend to swing around a hyperplane. Unlike the regression hyperplanes of memory accesses, the loop 
bounds hyperplane cannot be used directly. The regression hyperplane, by construction, minimizes the average square distance between the observed points. It implies that there could be points below and/or above the hyperplane. Consider the lower bound of a loop. Following the principles of construction of the lower bound regression hyperplane, there may be bounds that are actually above the computed hyperplane. If the loop is parallelized due to overapproximation, the generated code could scan points outside the actual domain. A similar situation occurs when the actual loop bounds are greater than the predicted hyperplane because this indicates that the generated code could skip some iterations that should be present in the domain. To handle this, for the lower bounding hyperplane, the hyperplane is shifted up by the width of the tube, with the rationale being that the shifted hyperplane acts as an expected minimum. Note that this still does not ensure that all the points are in the domain.

\section{The correlation is less than 0.9 .}

As with memory access regression hyperplanes, a correlation coefficient lower than 0.9 indicates that the regression is a poor fit for the observed loop bounds. However, some optimizing transformations can still be applied if a single value representing the lower bound can be found. Hence, a single value (rather than a hyperplane) indicating the predicted lower bound is selected from the observations.

Note that, unlike some existing schemes that handle a very simple outermost while loop [Collard 1995; Geuns et al. 2011; Griebl and Collard 1995; Lengauer and Griebl 1994], our system is capable of handling complex while loops appearing anywhere in the loop-nest. Also, in most of the existing schemes, only the upper limit is assumed to be unknown, whereas our system allows either upper bounds or lower bounds, or even both, to be unknown. There is also an additional challenge of handling nonlinear memory accesses along with nonlinear loop bounds.

If the nonlinear loop bound cannot be characterized by regression, and a minimal or maximal hyperplane cannot be constructed, or if after constructing the cutting hyperplanes the optimized part will only have a very low number of iterations, then the whole loop is subsumed to a statement such that the scheduler sees the whole loop as a single statement and processes accordingly.

\subsection{Scheduling and Skeleton Selection}

Once the dependence polyhedron has been constructed as just described, Pluto is used for scheduling. Traditionally, Pluto takes as input a ' $\mathrm{C}$ ' language-based code and produces a 'C' language-based output. However, in Apollo, Pluto is used as a library. Apollo directly encodes the dependence information into Pluto's internal structure and invokes its scheduler. The scheduler outputs the optimized schedule (in a matrix format). Note that, the Pluto's 'C' code generator (CLOOG) is not invoked by Apollo. In addition to the transformation, Apollo also identifies the loops that can be parallelized and uses this information to parallelize these loops. Based on the transformation properties, Apollo selects and instantiates an appropriate code skeleton.

\subsection{Safe Execution Point}

The optimizing transformations are deduced from the speculative prediction model that was built. To accommodate the possibility that the speculation may fail, the system must preserve a valid intermediate execution state, called a safe state. In the case of misspeculation, the safe state can be restored, and the original code execution can be restarted from this point. Without any safe intermediate state, the entire original code execution would have to be restarted from the very beginning of the target loopnest, which would induce a prohibitive time overhead and also would eliminate the opportunity of handling different dynamic phases of the same program. 
One way to construct a safe point would be to back up each memory location just before it is updated. This strategy can be implemented as (i) each thread backs up every location it is going to update or (ii) each thread communicates with others to know whether the location has already been backed up and the current thread backs up only if the other threads have not yet backed up the current location. However, (i) suffers from a huge amount of required memory because of the possible multiple copies. It also requires a mechanism to detect which backup occurred first among the multiple backups of the same location in order to be restored properly. On the other hand, (ii) requires thread synchronization and should be avoided for performance reasons. Finally, both strategies involve numerous 'malloc' calls, which obviously hurts performance.

Thanks to the linear functions, Apollo uses another more efficient technique for the backup. With the help of the linear functions characterizing the write memory accesses, and with the values of the loop bounds of the next launched chunk, Apollo can determine the memory write regions even before the chunk is launched. Note that, even in the case of misspeculation on linear functions, the latter is still valid because the memory accesses are guarded by the linear functions. Before launching the chunk, the predicted regions are backed up, thus avoiding the synchronization requirements and multiple copies. A main consequence is that Apollo guarantees that when no rollback has occurred, each frontier between consecutively executed chunks is a safe state. Nonlinear memory accesses and loop bounds introduce the additional challenge of handling the unpredicted memory writes.

In the case of nonaffine write accesses, there is no information in the model enabling prediction of the memory regions that will be read or written during the run of the next chunk. Hence, a "live" backup is performed during the speculative parallel execution of the next chunk in which each memory location is backed up just before being updated. For nearly affine write accesses, all the memory locations occurring inside the regression tube are backed up before launching the chunk. However, there still could be some outlier accesses occurring outside the predicted regression tubes; these, however, do not corrupt the semantics of the transformed loop nest. Since such accesses can only be known during the chunk execution, these accesses are handled similarly to the nonaffine case: They are backed up "live" during the chunk execution. As the live backup is performed by each thread in a distributed manner, one thread is not aware of the locations that may have been already backed up by another thread. Nevertheless, by using the prediction model, the amount of live backup is significantly reduced and therefore saves memory and execution time.

For loops with nonlinear bounds, the backup is computed based on the predicted minimum bound hyperplane and maximal bound hyperplane. Every location that may be updated between the hyperplanes is backed up before launching the chunk; those lying outside are backed up live by each thread, similarly to nonaffine write accesses.

Typical runtime speculation systems back up the data on the fly (live backup). Our experiments show that individually backing up memory locations is costly. This is shown in Figure 4, where different backup strategies are compared. An image processing kernel is studied to see the effect of different backup strategies while varying the number of nonlinear writes. Each nonlinear write corresponds to a write operation on one of the RGB components of the image. Each code version is named $\alpha$ _strategy, where $\alpha$ represents the number of nonlinear writes. In the "Runtime only" strategy, the whole backup is performed live (i.e., just before the write operation is performed). "Runtime only optimized" is an optimized version of "Runtime only" strategy, where the memory management is partially achieved by Apollo, thus reducing the number of required memory allocation calls. "Apollo combined" uses a two-pronged approach by using the prediction model. As explained earlier, by using the linear functions, 


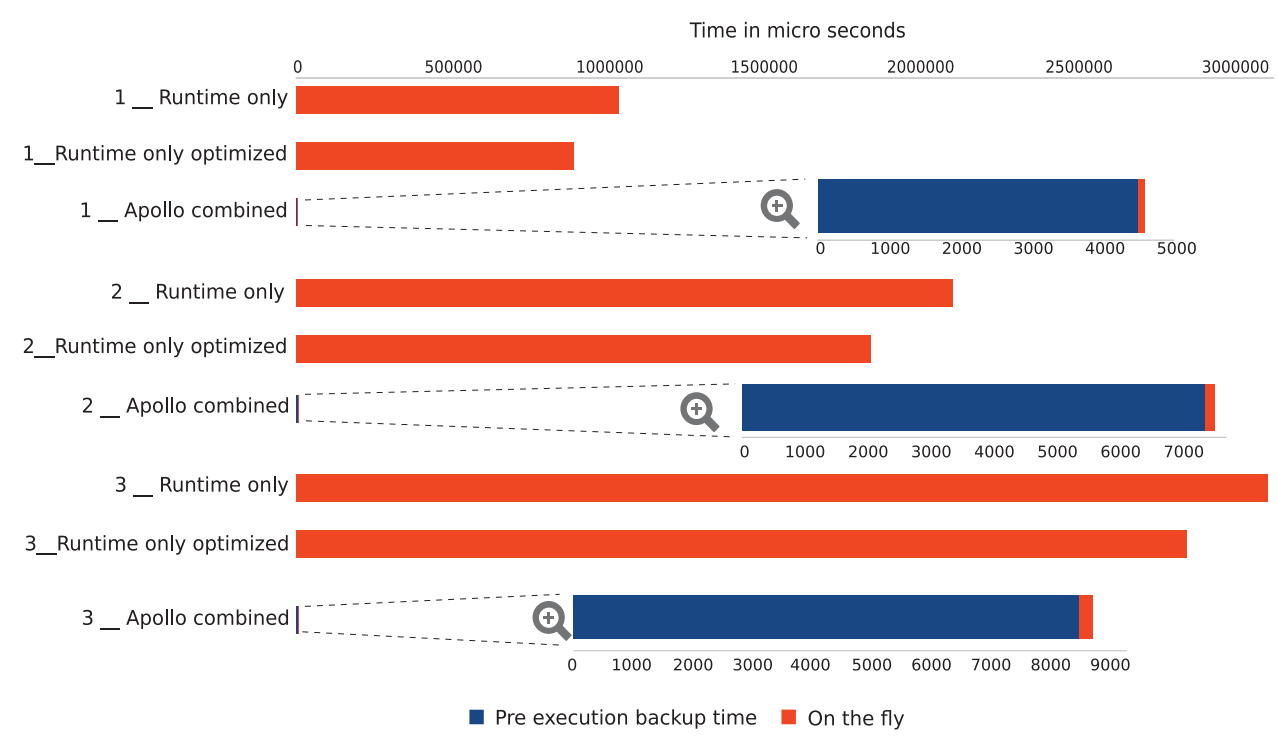

Fig. 4. Comparison of various backup strategies. Each code version is named $\alpha \_$strategy where $\alpha$ represents the number of nonlinear writes.

the regression functions, and the range information, Apollo tries to predict the set of memory locations that is going to be written during the run of the next chunk, and it backs up this region before the chunk is launched. Only the unpredicted accesses are backed up live. For the live backup, the memory management is also partially handled by Apollo, as in the "Runtime only optimized" strategy.

\subsection{Runtime Verification}

While the instantiated parallel skeleton runs inside a chunk, the runtime system must continuously ensure the correctness of the transformed code that is run by verifying (i) the adherence of the prediction model to the memory locations that are actually accessed and (ii) that occurrences of any unpredicted memory accesses do not invalidate the code transformation that has been applied regarding the code semantics.

The speculation verification system must be efficient to accommodate the fact that the verification is potentially associated with each memory instruction; at the same time, it must be general enough to handle all the different types of required verifications. The most common practice, especially in the field of thread-level speculation, is to use a centralized verification system [Johnson et al. 2007; Liu et al. 2006; Raman et al. 2008]. Such a system does not scale because each memory access may be subject to verification, which implies a huge time overhead induced by thread communication and synchronization. A comparison between centralized and decentralized verification approaches within Apollo is provided in Section 6. However, centralized verification, eager or lazy, cannot be avoided in all cases, especially when there are memory accesses that cannot be predicted. Nevertheless, the volume and frequency of communication to the central verification system can be greatly minimized by using an approximate prediction model. In Apollo, each thread verifies all its memory accesses in a decentralized manner, whereas only the unpredicted accesses whose impact cannot be disambiguated using the prediction model (and that potentially occur only rarely) are verified using a centralized mechanism. Moreover, notice that the centralized verification inside Apollo only occurs at the frontiers between consecutive chunks. 
The conformity of the predicted memory accesses regarding the predicted linear functions and the compliance of the unpredicted accesses to the speculative polyhedral model can only be verified during the actual execution of the optimized code. Each thread verifies the validity of each of its memory accesses against all the predicted memory accesses and thus performs an eager and decentralized verification. Depending on the accuracy of the model, the following verification operations are performed:

-If the memory instruction has been modeled exactly by an affine function, the thread checks the equality between the actual accessed address and the one predicted by instantiating the predicting affine functions. In case of misprediction, a potential risk of unpredicted dependence is detected;

-If the memory instruction has been modeled by a regression tube (or by a range of addresses), the thread verifies that the actual accessed address lies inside the tube (or inside the range). If not, the address is compared to the addresses that are predicted to be touched by all the other memory instructions during the execution of the current chunk. If no risk of interaction has been detected, the address is registered in a local table to be examined after the current chunk completion using the centralized verification. Otherwise, a potential risk of unpredicted dependence is detected.

If there is any potential risk of unpredicted dependence, a rollback is triggered by the faulty thread. Otherwise, at the end of the chunk execution, a cross-thread verification is performed to ensure cross-thread consistency by comparing the addresses that were registered in the respective local tables. The chances of this lazy verification failing is very small because the eager verifications performed by each thread detect, in most cases, any potential risk much earlier. However, if it fails, a rollback is triggered.

Detecting the possibility of a rollback as early as possible is crucial for performance. For the dynamically linear memory accesses and bounds, as soon as the interpolating linear functions have been constructed, the rest of the instrumented points are used for checking their correctness. If this fails, it is inferred that the program is not amenable to any transformation, at least in the current phase. For the nearly affine linear functions, the same principles are followed. For the nonaffine accesses and bounds, a simple and a fast check is done to verify if these accesses may interfere with any of the speculated affine or nearly affine accesses. Thus, this checking decreases the probability of rollback. Note that these checks are done before even launching any parallel chunk, thus saving the time overhead of memory backup, speculative execution, and rollback.

\subsection{Speculation Overhead Management}

Apollo evaluates the benefit of speculation by itself during the program run and thus is able to predict whether the speculation will provide any performance improvement. This self-check is very important when the number of speculative chunks that succeed are small, in which case the system should appropriately select the original code or the optimized one based on the predicted benefit. When a speculative chunk fails, it induces the following overheads: (i) the cost of backup, (ii) the time taken for running the (invalid) optimized skeleton, (iii) the time to rollback, and (iv) the time required to run the original code to overcome the faulty chunk. To analyze the cost of rollbacks, consider Figure 5, which profiles an image processing code kernel where the number of nonlinear write accesses is controlled. One nonlinear write represents a write to one of the three RGB components of the image. In addition to the number of nonlinear writes, the number of rollbacks is also controlled by forcing Apollo to rollback.

In the figure, only the time lost by misspeculation is measured. The backup time represents the time required to compute the backup region as well as the cost of memory allocation and data copying. It also includes the cost of live backup. The 


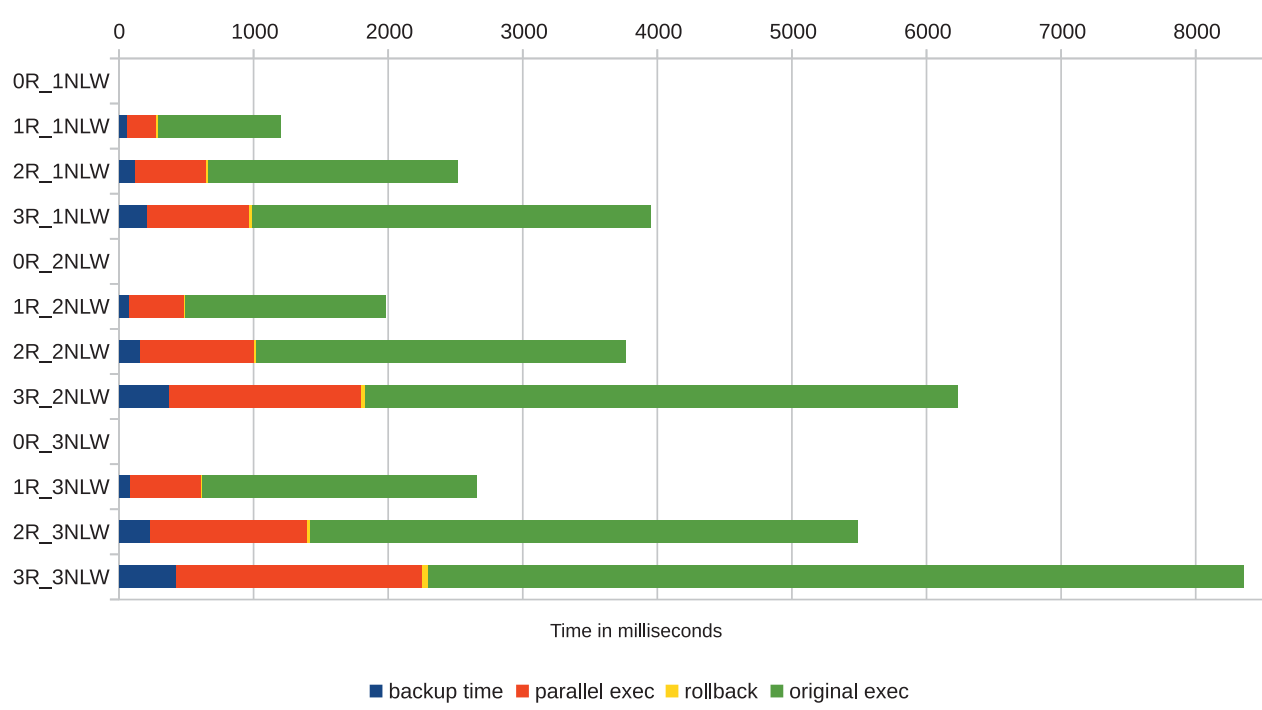

Fig. 5. Overhead of misspeculation. Each code version is name $\alpha \mathrm{R} \_\beta \mathrm{NLW}$ where $\alpha$ represents the number of rollbacks and $\beta$ represents the number of nonlinear writes.

parallel execution time represents the time spent running the optimized skeleton. The rollback represents the time required to restore memory, while the original execution time represents the time taken by the original skeleton to re-run the misspeculated chunk. Note that since the figure only represents the time lost in the case of a misspeculation, the values are zero when there is no misspeculation. As expected, the total cost of the rollbacks approximately follows a linear relationship with that of a single rollback. Furthermore, the cost obviously increases as the number of nonlinear writes are increased.

It is obvious that performance degradation occurs when the system encounters multiple rollbacks. In Apollo, if the speculation fails consecutively for the first three chunks, the rest of the execution completes using the original code. However, if the system encounters a mix of speculation successes and failures, it predicts the possible performance gain of a single chunk, and, following this prediction, the original or parallel skeleton is selected. This is done as follows: When a speculative chunk fails, Apollo measures the individual time for backup, parallel execution, rollback, and original execution. Note that even though the computation was wrong (because speculation failed), the parallel execution time approximately remains the same as that of a correct computation. To obtain an interesting speedup, the ratio original sequential executiontime $(T O) /$ apollo optimizedexecutiontime $(T A)$ should be greater than 1.

Let $f$ denote the number of chunks that failed and $p$ denote the number of chunks that succeeded. Let $F$ denote the total time required for executing and recovering a failed chunk, $P$ denote the time required for executing a parallel chunk along with the associated backup, $O$ denote the execution time of an original chunk, and $R$ denote the restore time. Let $\gamma$ denote the expected speedup of an optimized chunk when compared to the original chunk (i.e., $\gamma=O / P$ ). We want the execution time of Apollo to be less than the execution time of the original serial code; that is:

$$
T O / T A \geq 1
$$


where:

$$
\begin{gathered}
T A=p * P+f * F \\
T O \approx(p+f) * O .
\end{gathered}
$$

The execution time of a failed chunk consists of the parallel execution time (which includes the backup time), the rollback time, and the original serial execution time. The rollback time is significantly less than the parallel execution time and the original time and thus can be ignored:

$$
F=P+R+O \approx P+O .
$$

By substituting Equations (2), (3), and (4) in Equation (1) and rearranging, we get:

$$
p * O+f * O \geq p * P+f * P+f * O .
$$

By substituting $\gamma=O / P$ in Equation (5), removing $f * O$ on both sides, and rearranging, we obtain:

$$
(\gamma-1) \geq f / p
$$

Equation (6) relates the expected speedup to the number of chunks that can speculatively succeed or fail. Apollo uses the latter to infer the effectiveness of speculating in cases where there is a mix of succeeding and failing chunks, and it selects the skeletons accordingly.

\subsection{Putting It All Together}

Given an input program and target loop nests marked by the "apollo pragma," Apollo statically analyzes the loop nest and produces a set of generic code skeletons that represent some generic combinations of polyhedral transformations. At runtime, Apollo first profiles the code by employing instrumentation by sampling. Based on the profile, Apollo computes the linear equations, regression equations, and ranges suitable for the modeling of each memory access and loop bound, as explained in Section 4.1; these are then encoded into a relaxed polyhedral model. Once the dynamic polyhedral model has been constructed, the polyhedral scheduler Pluto is invoked to produce an optimizing parallelizing transformation. Based on the dynamic polyhedral model, a safe point is computed by backing up the predicted memory write regions, and the speculative chunk is launched. The speculative chunk is monitored constantly, in a decentralized manner, to detect any potential dependence violation. If an unpredicted memory access occurs inside a speculative chunk, live data backup is performed. If the speculative chunk completes successfully, a cross-thread verification, which validates the program consistency across threads, is launched. The probability of this verification failing is low because the distributed verification, in most cases, detects any potential violation much earlier. If the speculative execution succeeds, the speculative transformation is reapplied for the next chunk and so on. On the other hand, if the speculation fails, a rollback is performed, and a chunk executing the original serial code is launched, which is then followed again by an instrumentation phase if speculation has still been evaluated as beneficial. The whole process is continued until the entire target loop nest has been executed.

\section{RELATED WORK}

TLS systems are the main alternatives when it comes to automatically parallelizing loops that are not fully analyzable during compilation. They generally apply a straightforward parallelization strategy based on slicing the outermost loop into parallel threads while implementing various speculation validation strategies. 
Oacean, Mycroft, and Harris [Oancea et al. 2009] propose an in-place TLS system. They do not perform any predictions or transformations of the code. Checking for new dependences is performed on every memory access. Any cross-iteration dependency causes a rollback.

The approach implemented in the SAMBAMBA framework [Streit et al. 2013] uses a software transactional memory framework (TinySTM) to handle dependency violations among threads. The authors do not apply polyhedral transformations.

The work presented in Raman et al. [2010] is a software transactional memory system that aims to perform better in the context of thread-level speculation. Memory updates are handled by means of a centralized commit unit, which is decoupled from the conflict checking. For the latter, no major solution is proposed.

Some previous work looks at handling while loops in a limited setting. The paper from Martin Griebl and Collard [1995] proposes a method to handle while loops using the polyhedral model. However, the approach does not allow pointers or pointer-based accesses and requires all the accesses to be affine. It also requires a centralization mechanism that needs synchronization in each and every iteration of the loop nest. The impact on performance is not studied. Our comparison with a centralized system in Section 6 shows that our system performs better even with the added support for nonaffine accesses. Another work by Jean-Francois Collard [1995] adds a very basic speculative support to the latter; however, the work further restricts the scope to programs with a single outermost while loop. The work requires huge amounts of memory, and all the latter restrictions apply. In Geuns et al. [2011], Stefan J. Geuns et al. propose a framework to extract task-based parallelism. Polyhedral transformations cannot be applied using this approach, and the system is focused on a very specific application. The work tries to parallelize while loops by extracting task-based parallelization. Each task represented by a node is task graph, and the shared variables form the edges. Special shared data structures are created to share variables. In addition to this, the work requires the memory accesses to follow dynamic single assignment per section (in a code section, the memory writes be unique), which limits the scope of the work. Our system is able to parallelize and apply advanced loop transformations, and we do not rely on dynamic single assignment. Moreover, the shared data structures proposed in the wor have limited size, thereby limiting parallelism, whereas we have no such restrictions. The work in Lengauer and Griebl [1994] follows a similar approach to that of Collard [1995] but chooses a conservative approach instead of speculation; no information regarding the applicability or the performance impact is available.

PPCG [Verdoolaege et al. 2013] is a polyhedral compiler targeting statically analyzable code for GPU processors. In PPCG, dynamic control statements are supported by subsuming the whole loop that contains the nonaffine control to a single statement (i.e., if the loop contains a dynamic control flow (break, continue, etc.) while building the polyhedral model, the whole loop is represented as a single statement). This clearly limits the optimization opportunities. For example, in this scheme, a while loop or a for loop containing a dynamic control flow can never be parallelized. Moreover, the memory accesses must always be statically affine.

Collard et al. [1995] present a static analysis procedure to find approximate solutions to dataflow problems involving unknowns such as the iteration count of a while statement or nonaffine array subscripts. Pointers and aliasing are not allowed in the handled programs. Moreover, since it is static, the approach is necessarily conservative, whereas our runtime approach handles the actual memory references and iteration counts that yield obviously more accurate approximations.

Anand Venkat et al. [2014] propose a method to handle nonlinear codes using the polyhedral model. It only handles sparse matrix computations where the nonlinear memory accesses that are considered are exclusively read operations through 
indirections. It is based on an inspector-executor mechanism. During the inspector phase, it collects all the nonlinear memory reads and creates a mapping function that maps the iteration space to the nonlinear memory access addresses. The executor then uses the inverse of the created function to re-map the addresses in the transformed space. When compared to our approach, this approach has the following major limitations: (i) The work only supports nonlinear reads, whereas our work also supports writes, which are crucial regarding data dependences. (ii) The work relies on an inspector-executor mechanism. Thus, as with any inspector-executor system, it can only handle codes where the computation of the target memory addresses can be split from the accesses. Many codes violate this assumption, especially when using pointers. (iii) It is the responsibility of the user to ensure that the nonlinear reads are disjoint from the write regions. Hence, no related dependence analysis is performed by the proposed system. (iv) Since the inspector code is sequential, its associated overhead has to be amortized over many calls to the target loop nest. In our proposal, each run of a target loop nest is handled and optimized. Thus, when compared to our system, the proposed system has less automation.

In comparison, Apollo features a linear prediction model using interpolation and regression; applies optimizing polyhedral transformation on-the-fly, aiming for automatic data locality optimization and parallelization; and takes advantage of a hybrid centralized/decentralized speculation verification system that significantly reduces the time overhead of data race detection. Another key aspect of the proposed system is that it handles pointers and nonaffine accesses along with nonaffine loop bounds.

\section{RESULTS}

The experiments were run on a platform embedding two AMD Opteron 6172 processors of 12 cores each, at $2.1 \mathrm{Ghz}$, running Linux 3.11.0-17-generic x86_64. The reported measurements were obtained by running each benchmark five times and taking their average. The speedups are reported against the best performing serial codes among clang and gcc-compiled binaries with flag '-O3'.

The benchmarks were selected from various benchmark suites such as the SPARK00 benchmark suite [van der Spek et al. 2008] (trmat, ispmatmat, ispmatvec, mperm), the Rodinia benchmark suite [Che et al. 2009] (srad, b+tree), Cbench [Fursin and Temam 2010] (automotive_susan_c, automotive_susan_e), and the Perfect benchmark suite [Barker et al. 2013] (pfa-interp1, backprojection). They were selected based on their loop properties and memory access patterns. It is worth noting that these benchmarks cannot be optimized by tools like Pluto or the previous version of Apollo due to the nonlinearity of the memory accesses and loop bounds. In addition to these benchmarks, we included Filter ${ }^{3}$, a real-world image processing application that exhibits nonlinear array references. Every benchmark source code is written in $C / C++$.

To show the gains provided by Apollo compared to a standard TLS system with centralized speculation verification, we created a modified version of our framework to simulate such a centralized mechanism. In the centralized verification system, each thread has to communicate with each other. Our modification only consists of a call to a runtime function for each memory access that the loop attempts to perform, thus simulating such a centralized invocation. Inside the centralized runtime verification function, two values are added and the result is written to a memory location. No other operations are performed. Note that these operations are the bare minimum for a centralized system. Furthermore, in our centralized version, the outermost loop is parallelized and no memory backup is performed. We would like to emphasize the fact that, in this version, since the runtime verification simply performs a write, not

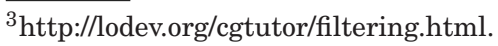




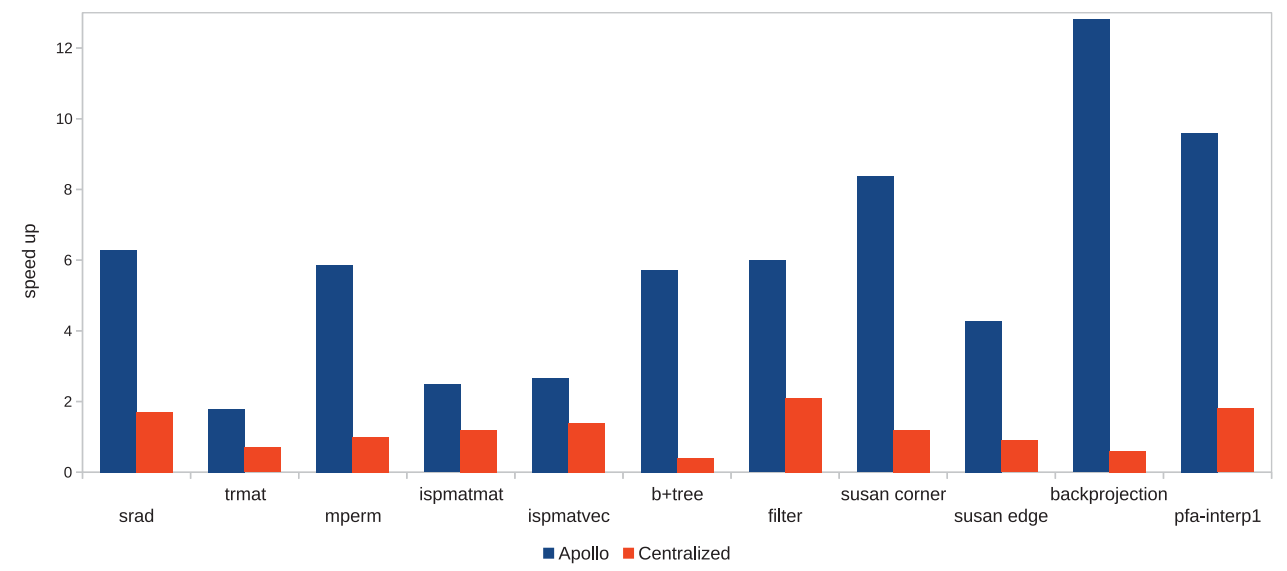

Fig. 6. Speedup of nonlinear Apollo and the centralized system, using 24 threads.

even the overhead of dependence verification is present and the dependencies are always assumed to be respected. Thus, all actions are chosen in favor of the centralized mechanism (no backup, no dependence checking, no central checks). The addresses of the write locations were carefully chosen so that they reside in different cache lines to avoid the penalty of cache coherence protocols. This measurement serves as a base for comparing against centralized systems, since this modified version only performs a minimum amount of operations per memory access.

A real centralized system would have to perform costlier operations and would rely on a central algorithm. It should be emphasized that our system is based on a weakly centralized model, having a very small centralized verification in addition to possible live backups. However, the weakly centralized verification is not live and hence does not cause bottlenecks. Also, as mentioned earlier, the chances for a rollback occuring at this step are very low thanks to our prediction model, nonlinear analysis, and eager verification system.

In Figure 6, we show the speedups of Apollo using the nonlinear modeling and verification system that were presented. As mentioned earlier, we compare our framework to the centralized verification system. For all the benchmarks, Apollo outperforms the centralized version while providing significant speed-ups.

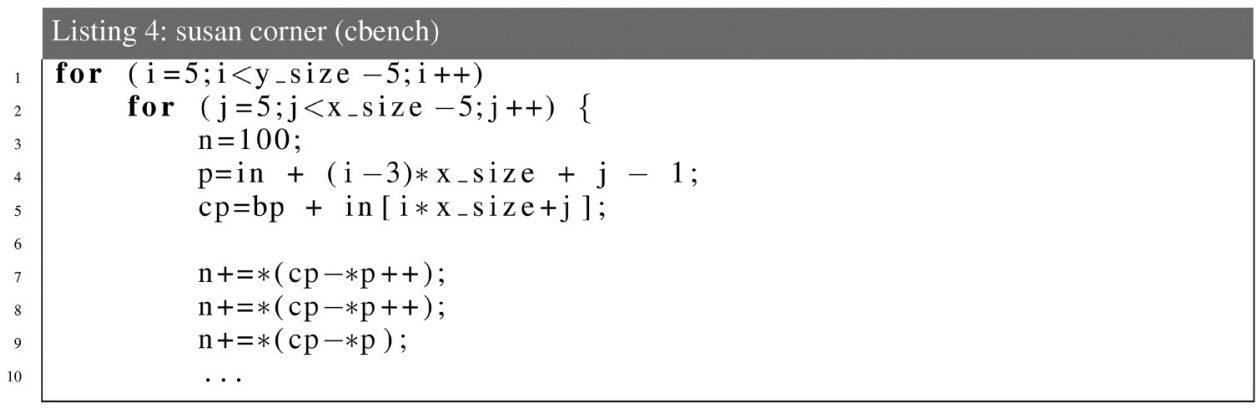

Benchmark trmat contains nonlinear loop bounds and nonlinear memory accesses (as explained in Section 2.3) that are modeled using regression tubes. Listing 4 shows an extract of the susan corner detection kernel from the Cbench benchmark suite. Lines 6 , 7, 8 (and a long list of lines not shown here) access a memory location whose address is the result of a pointer computation depending on 'cp', which is updated in Line 5. The 
computation of 'cp' only uses linear memory accesses. However, its successive values are not linear. Since it is used for an address computation, the resulting address is not linear. Range modeling was used for this code because the regression coefficient was low. Benchmarks ispmatmat and ispmatvec are both modeled using regression tubes. Benchmark pfa-interp1, shown in Listing 5 (only some parts are shown), has nonlinear conditionals, nonlinear loops, and nonlinear memory accesses. Line 5 assigns the result of a function to variable 'nearest', which is then used in the if-condition in Line 7, which leads to a dynamic nonlinear conditional. Lines 13, 15, 16, and 18 update variables rmin and rmax, which are then used as loop bounds for the for-loop in Line 19. Since these values are not linear, the loop bounds are also not linear and, consequently, neither are the memory accesses in Lines 20,24 and 25. This benchmark is handled using the range-based mechanism. In a nutshell, regression tubes were used to model codes srad, trmat, ispmatmat, ispmatvec, filter, and backprojection, whereas ranges were used to model codes mperm, b+tree, susan corner, susan edge, and pfa-interp1.

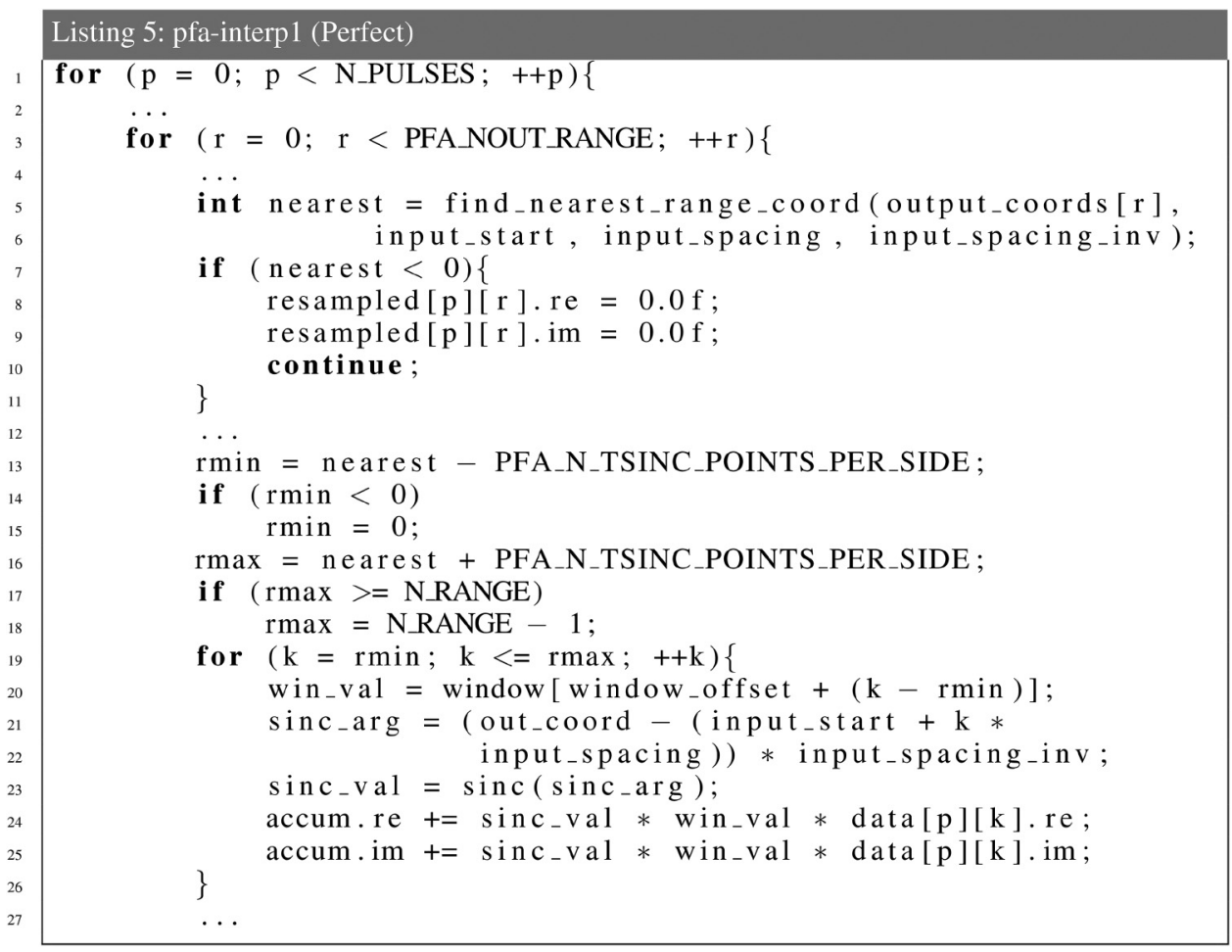

Figure 7 shows a classification regarding the time overhead of the system. Only the major overheads are considered. The instrumentation time refers to the time taken for code profiling. It includes the time for running a small chunk and the time to register the memory accesses and loop bounds information to the runtime. The solving access functions refers to the time spent on resolving the memory accesses and loop bounds interpolating functions. It also includes the time for finding the regression functions, the range functions, and the regression coefficients, which classifies the access as affine, nearly affine, or nonaffine. The scheduling time refers to the time taken by the scheduler (i.e., the time taken to instantiate the scheduler and the time taken by Pluto's scheduler to determine an optimal schedule). The FM time refers to the time required for Fourier-Motzkin elimination. Fourier-Motzkin elimination is used to 


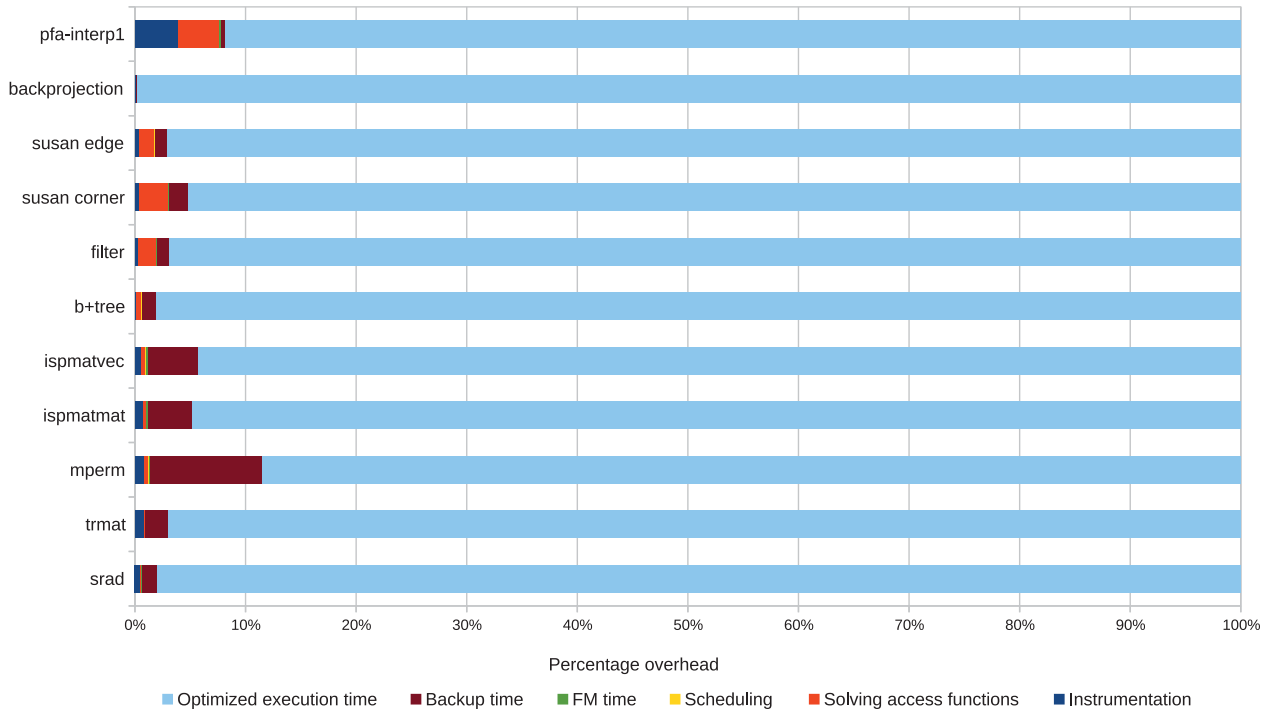

Fig. 7. Overhead classification in percentage.

Table II. Comparison of Binary Sizes

\begin{tabular}{|c|c|c|c|c|}
\hline \multirow[b]{2}{*}{ Benchmark } & \multicolumn{2}{|c|}{ Apollo (in KB) } & \multirow{2}{*}{$\begin{array}{c}\text { clang } 3.4 \\
\text { (in KB) }\end{array}$} & \multirow{2}{*}{$\begin{array}{c}\text { gcc } 4.8 .2 \\
\text { (in } \mathrm{KB} \text { ) }\end{array}$} \\
\hline & Binary size & Runtime (shared lib) & & \\
\hline trmat & 140.7 & 566.7 & 23.6 & 28.1 \\
\hline mperm & 153 & 566.7 & 27.8 & 28.1 \\
\hline ispmatmat & 177.6 & 566.7 & 23.7 & 28.1 \\
\hline ispmatvec & 140.7 & 566.7 & 23.7 & 23.9 \\
\hline $\mathrm{b}+$ tree & 228.9 & 566.7 & 38.3 & 42.4 \\
\hline filter & 200 & 566.7 & 13.2 & 9 \\
\hline srad & 152.2 & 566.7 & 17.6 & 17.5 \\
\hline susan corner & 342.6 & 566.7 & 43.2 & 43.2 \\
\hline susan edge & 395.8 & 566.7 & 43.2 & 43.2 \\
\hline backprojection & 145.5 & 566.7 & 18.2 & 18.4 \\
\hline pfa-interp1 & 160.8 & 566.7 & 18.1 & 18.6 \\
\hline
\end{tabular}

determine the loop bounds in the transformed iteration space. If the transformation remains the same across contiguous chunks, the FM solver is only invoked once, but the resulting functions are reused for each chunk. However, if the transformation is changed, the FM solver is invoked again. Backup time refers to the time taken by the backup system to calculate the memory area that needs to be backed up and the time for actual backup. The backup consists of copying regions of (i) affine memory accesses (computed using affine functions), (ii) nearly affine accesses (computed using the tube formulation of regression functions), and (iii) nonaffine memory accesses (predicted using range information). The optimized skeleton refers to the time spent on actual parallel execution, decentralized verification, and live backup.

Table II compares the sizes of the binaries generated by Apollo against the sizes of the binaries generated by compilers gcc and clang. On average, the binary size produced by Apollo is 6.7 times larger than that of clang and 6.4 times larger than that of gcc. The increase in size is due to the presence of multiple skeletons. Also, each skeleton in LLVM-IR is encoded inside the binary, which is later compiled at runtime using the 
LLVM JIT compiler. The runtime is compiled as a Shared Object (SO) file. Apollo also requires some standard LLVM libraries for the JIT.

\section{CONCLUSION}

The polyhedral model is a well-established mathematical model used to optimize and parallelize loop nests. The applicability of the model on codes with dynamically polyhedral or nonaffine behavior was impossible due to unavailable crucial information during static compilation. We have shown that, thanks to a dynamic and speculative approach and by relaxing the required constraints, applicability of the polyhedral model can be extended to codes with dynamic behavior that do not naturally fit to the model. This work highlights effective solutions on how to relax certain constraints and how to account for code correctness by using runtime verification that induces low time overhead. Thus, this work effectively increases the domain of codes that can take advantage of complex optimizing transformations at runtime to include those codes that exhibits nonlinear memory behaviors. As shown in the experiments, aggressive speculative polyhedral transformations, including parallelization-along with our verification system, which handles most of the verification in a distributed manner-can yield very interesting speedups. Moreover, Apollo also highlights the fact that codes may exhibit interesting optimization opportunities depending on the processed input. This work opens the path to investigations related to new memory allocation and access strategies that may be better adapted to code parallelization and optimization, either in software or hardware.

\section{REFERENCES}

U. Banerjee. 1993. Loop Transformations for Restructuring Compilers - The Foundations. Kluwer Academic Publishers.

Kevin Barker, Thomas Benson, Dan Campbell, David Ediger, Roberto Gioiosa, Adolfy Hoisie, Darren Kerbyson, Joseph Manzano, Andres Marquez, Leon Song, Nathan Tallent, and Antonino Tumeo. 2013. PERFECT (Power Efficiency Revolution for Embedded Computing Technologies) Benchmark Suite Manual. Pacific Northwest National Laboratory and Georgia Tech Research Institute. http://hpc. pnnl.gov/projects/PERFECT/.

Emery D. Berger and Benjamin G. Zorn. 2006. DieHard: Probabilistic memory safety for unsafe languages. In Proceedings of the 27th ACM SIGPLAN Conference on Programming Language Design and Implementation (PLDI'06). ACM, New York, NY, USA, 158-168. DOI : http://dx.doi.org/10.1145/1133981.1134000

Uday Bondhugula, Albert Hartono, J. Ramanujam, and P. Sadayappan. 2008. A practical automatic polyhedral parallelizer and locality optimizer. In Proceedings of the 29th ACM SIGPLAN Conference on Programming Language Design and Implementation (PLDI'08). ACM, New York, NY, USA, 101-113. DOI : http://dx.doi.org/10.1145/1375581.1375595

Shuai Che, Michael Boyer, Jiayuan Meng, David Tarjan, Jeremy W. Sheaffer, Sang-Ha Lee, and Kevin Skadron. 2009. Rodinia: A benchmark suite for heterogeneous computing. In IISWC. IEEE, 44-54.

Jacob Cohen, Patricia Cohen, Stephen G. West, and Leona S. Aiken. 2002. Applied Multiple Regression / Correlation Analysis for the Behavioral Sciences (3rd ed.). Routledge.

Jean-François Collard. 1995. Automatic parallelization of while-loops using speculative execution. International Journal of Parallel Programming 23, 2 (April 1995), 191-219. DOI:http://dx.doi.org/10. 1007/BF02577789

Jean-François Collard, Denis Barthou, and Paul Feautrier. 1995. Fuzzy array dataflow analysis. SIGPLAN Not. 30, 8 (Aug. 1995), 92-101. DOI : http://dx.doi.org/10.1145/209937.209947

Paul Feautrier and Christian Lengauer. 2011. Polyhedron model. In Encyclopedia of Parallel Computing, David Padua (Ed.). Springer US, 1581-1592. DOI :http://dx.doi.org/10.1007/978-0-387-09766-4_502

Grigori Fursin and Olivier Temam. 2010. Collective optimization: A practical collaborative approach. ACM Transactions on Architecture and Code Optimization 7, 4, Article 20 (Dec. 2010), 29 pages. DOI:http:// dx.doi.org/10.1145/1880043.1880047

Stefan J. Geuns, Marco J. G. Bekooij, Tjerk Bijlsma, and Henk Corporaal. 2011. Parallelization of while loops in nested loop programs for shared-memory multiprocessor systems. In Design, Automation \& Test in Europe Conference \& Exhibition, DATE 2011. IEEE Computer Society, 1-6. http://doc.utwente.nl/78154/ 
Martin Griebl and Jean-Francois Collard. 1995. Generation of synchronous code for automatic parallelization of while loops. In Proceedings of the Euro-Par'95 Parallel Processing, First International Euro-Par Conference, Stockholm, Sweden, August 29-31, 1995. 315-326. DOI : http://dx.doi.org/10.1007/BFb0020474

Alexandra Jimborean, Philippe Clauss, Jean-François Dollinger, Vincent Loechner, and Martinez Juan Manuel. 2014. Dynamic and speculative polyhedral parallelization using compiler-generated skeletons. International Journal of Parallel Programming 42, 4 (Aug. 2014), 529-545.

Troy A. Johnson, Rudolf Eigenmann, and T. N. Vijaykumar. 2007. Speculative thread decomposition through empirical optimization. In Proceedings of the 12th ACM SIGPLAN Symposium on Principles and Practice of Parallel Programming (PPoPP'07). ACM, New York, NY, USA, $205-214$. DOI : http://dx.doi.org/10.1145/1229428.1229474

Christian Lengauer and Martin Griebl. 1994. On the Parallelization of Loop Nests Containing While Loops. Technical Report MIP-9414. Universitt Passau (DE). http://opac.inria.fr/record=b1040396

Wei Liu, James Tuck, Luis Ceze, Wonsun Ahn, Karin Strauss, Jose Renau, and Josep Torrellas. 2006. POSH: A TLS compiler that exploits program structure. In Proceedings of the Eleventh ACM SIGPLAN Symposium on Principles and Practice of Parallel Programming (PPoPP'06). ACM, New York, NY, USA, 158-167. DOI : http://dx.doi.org/10.1145/1122971.1122997

Gene Novark and Emery D. Berger. 2010. DieHarder: Securing the heap. In Proceedings of the 17th ACM Conference on Computer and Communications Security (CCS'10). ACM, New York, NY, USA, 573-584. DOI : http://dx.doi.org/10.1145/1866307.1866371

Cosmin E. Oancea, Alan Mycroft, and Tim Harris. 2009. A lightweight in-place implementation for software thread-level speculation. In Proceedings of the Twenty-first Annual Symposium on Parallelism in Algorithms and Architectures (SPAA'09). ACM, New York, NY, USA, $223-232$. DOI : http://dx.doi.org/10.1145/1583991.1584050

Arun Raman, Hanjun Kim, Thomas R. Mason, Thomas B. Jablin, and David I. August. 2010. Speculative parallelization using software multi-threaded transactions. In ACM SIGARCH Computer Architecture News, 38, 1 (March 2010), 65-76. DOI : http://dx.doi.org/10.1145/1735970.1736030

Easwaran Raman, Neil Va hharajani, Ram Rangan, and David I. August. 2008. Spice: Speculative parallel iteration chunk execution. In Proceedings of the 6th Annual IEEE/ACM International Symposium on Code Generation and Optimization (CGO'08). ACM, New York, NY, USA, 175-184. DOI : http://dx.doi.org/10.1145/1356058.1356082

Lawrence Rauchwerger and David Padua. 1995. The LRPD test: Speculative run-time parallelization of loops with privatization and reduction parallelization. In Proceedings of the ACM SIGPLAN 1995 Conference on Programming Language Design and Implementation (PLDI'95). ACM, New York, NY, USA, 218-232. DOI : http://dx.doi.org/10.1145/207110.207148

Mahesh Ravishankar, John Eisenlohr, Louis-Noël Pouchet, J. Ramanujam, Atanas Rountev, and P. Sadayappan. 2012. Code generation for parallel execution of a class of irregular loops on distributed memory systems. In Proceedings of the International Conference on High Performance Computing, Networking, Storage and Analysis (SC'12). IEEE Computer Society Press, Los Alamitos, CA, USA.

Kevin Streit, Clemens Hammacher, Andreas Zeller, and Sebastian Hack. 2013. Sambamba: Runtime adaptive parallel execution. In Proceedings of the 3rd International Workshop on Adaptive Self-Tuning Computing Systems (ADAPT'13). ACM, New York, NY, USA, Article 7, 6 pages. DOI:http://dx.doi.org/ $10.1145 / 2484904.2484911$

Aravind Sukumaran-Rajam, Juan Manuel Martinez, Willy Wolff, Alexandra Jimborean, and Philippe Clauss. 2014. Speculative program parallelization with scalable and decentralized runtime verification. In Runtime Verification, Borzoo Bonakdarpour and Scott A. Smolka (Eds.), Vol. 8734. Springer, Toronto, Canada, 124-139. DOI : http://dx.doi.org/10.1007/978-3-319-11164-3_11

Harmen L. A. van der Spek, Erwin M. Bakker, and Harry A. G. Wijshoff. 2008. SPARK00: A benchmark package for the compiler evaluation of irregular/sparse codes. CoRR abs/0805.3897 (2008).

Anand Venkat, Manu Shantharam, Mary Hall, and Michelle Mills Strout. 2014. Non-affine extensions to polyhedral code generation. In Proceedings of Annual IEEE/ACM International Symposium on Code Generation and Optimization (CGO'14). ACM, New York, NY, USA, Article 185, 10 pages. DOI : http://dx.doi.org/ $10.1145 / 2544137.2544141$

Sven Verdoolaege, Juan Carlos Juega, Albert Cohen, José Ignacio Gómez, Christian Tenllado, and Francky Catthoor. 2013. Polyhedral parallel code generation for CUDA. ACM Transactions on Architecture and Code Optimization 9, 4, Article 54 (Jan. 2013), 23 pages. DOI : http://dx.doi.org/10.1145/2400682.2400713

Received May 2015; revised October 2015; accepted October 2015 